\title{
Pre-implantation alcohol exposure induces lasting sex-specific DNA methylation programming errors in the developing forebrain
}

\author{
L. M. Legault ${ }^{1,2}$, K. Doiron ${ }^{1}$, M. Breton-Larrivée ${ }^{1,2}$, A. Langford-Avelar ${ }^{1,2}$, A. Lemieux ${ }^{1,2}$, M. Caron¹, \\ L. A. Jerome-Majewska ${ }^{3,4}$, D. Sinnett ${ }^{1,5}$ and S. McGraw ${ }^{1,2,6^{*}}$ (D)
}

\begin{abstract}
Background: Prenatal alcohol exposure is recognized for altering DNA methylation profiles of brain cells during development, and to be part of the molecular basis underpinning Fetal Alcohol Spectrum Disorder (FASD) etiology. However, we have negligible information on the effects of alcohol exposure during pre-implantation, the early embryonic window marked with dynamic DNA methylation reprogramming, and on how this may rewire the brain developmental program.
\end{abstract}

Results: Using a pre-clinical in vivo mouse model, we show that a binge-like alcohol exposure during pre-implantation at the 8-cell stage leads to surge in morphological brain defects and adverse developmental outcomes during fetal life. Genome-wide DNA methylation analyses of fetal forebrains uncovered sex-specific alterations, including partial loss of DNA methylation maintenance at imprinting control regions, and abnormal de novo DNA methylation profiles in various biological pathways (e.g., neural/brain development).

Conclusion: These findings support that alcohol-induced DNA methylation programming deviations during preimplantation could contribute to the manifestation of neurodevelopmental phenotypes associated with FASD.

Keywords: Prenatal exposure, Fetal alcohol spectrum disorder, DNA methylation, Early embryonic development, Epigenetic reprogramming, Imprinting

\section{Introduction}

Fetal Alcohol Spectrum Disorder (FASD) encompasses the range of lifelong cognitive and physical disabilities observed in children born to mothers who consumed alcohol during pregnancy [1-4]. Each year, 600,000-1 million children are born with FASD worldwide $[5,6]$. The most severe and physically visible form of the condition is known as Fetal Alcohol Syndrome (FAS), in $\sim 10 \%$ of FASD cases, and associated with the full presentation of dysmorphic features including craniofacial

*Correspondence: serge.mcgraw@umontreal.ca

${ }^{1}$ CHU Sainte-Justine Research Center, 3175 Chemin de La

Côte-Sainte-Catherine, Montréal, QC H3T 1C5, Canada

Full list of author information is available at the end of the article malformations, growth deficits and structural brain pathologies. Depending on the amount, pattern and developmental period of prenatal alcohol exposure, children may not present with dysmorphic features, but still suffer from mild to severe FASD-related neurological disabilities, such as learning deficits and intellectual delays $[1,2]$. With the marked increase in rates of alcohol use and binge drinking behavior [7] among 18-34 year old women [8-14], added to the high number of unintended pregnancies worldwide $(\sim 40 \% ; 85$ million/year [15]), many women may inadvertently subject their developing embryos to acute levels of alcohol in the first weeks of pregnancy. Although most studies state that alcohol consumption at all stages of pregnancy can cause FASD, preimplantation is arguably the stage that is most prone to original author(s) and the source, provide a link to the Creative Commons licence, and indicate if changes were made. The images or other third party material in this article are included in the article's Creative Commons licence, unless indicated otherwise in a credit line to the material. If material is not included in the article's Creative Commons licence and your intended use is not permitted by statutory regulation or exceeds the permitted use, you will need to obtain permission directly from the copyright holder. To view a copy of this licence, visit http://creativecommons.org/licenses/by/4.0/. The Creative Commons Public Domain Dedication waiver (http://creativeco mmons.org/publicdomain/zero/1.0/) applies to the data made available in this article, unless otherwise stated in a credit line to the data. 
unintentional prenatal alcohol exposure since the human chorionic gonadotrophin (hCG) hormone, the main biomarker for pregnancy, is not yet detectable as it is only produced following implantation. Still, there is ample misinformation in the literature regarding the effects of alcohol exposure, and many other teratogen exposures, on pre-implantation embryos, and how this leads to an "all-or-nothing" developmental outcome.

An increasing body of evidence indicates that alcohol exposure during fetal brain development triggers lasting epigenetic alterations, including DNA methylation, in offspring long after the initial insult, supporting the role of epigenetics in FASD phenotypes [16-19]. However, we remain unaware of how ethanol affects the early developmental window marked with dynamic changes in DNA methylation, and how interfering with this fundamental process may program future FASD-related neurological disabilities. During pre-implantation development, the period between oocyte fertilization and embryo implantation in the uterus, the epigenome undergoes a broad reprogramming that initiates the developmental program [20-26]. We and others have shown that this essential reprogramming wave removes most DNA methylation signatures across the genome, except specific sequences that include imprinting control regions (ICRs), to trigger the embryonic developmental program [20, 27-29]. DNA methylation marks are then reacquired in a sex-, cell- and tissue-specific manner during the peri-implantation period, and marks continue to be modulated during lineage specification [30-33]. Studies show that pre-implantation embryos can have sex-specific epigenetic responses to similar environmental challenges, leading to long-term sexual dimorphism in developmental programming trajectories [34-37].

One of the first indications of the direct link between ethanol exposure and aberrations in DNA methylation came from a mouse study showing that mid-gestation exposure at E9-E11 reduced global DNA methylation levels in E11 fetuses [17]. This evidence gave rise to different FASD models using various levels of short or prolonged alcohol exposures at different stages of gestation. Ethanol exposure can either induce DNA methyltransferases (DNMTs) activity, through reactive oxygen species-dependent mechanisms [38, 39], or inhibit DNMTs activity, via direct action on DNMTs or on one-carbon metabolism that provides methyl groups [17, 40], which supports why both gain and loss of DNA methylation marks can be observed in FASD models. High levels of ethanol exposure on two consecutive days (E1.5, E2.5) altered DNA methylation of imprinted gene $H 19$, a negative regulator of growth and proliferation, in the placenta at E10.5, yet this region showed no alteration in the embryo [41]. Nonetheless, data remain very scarce on how alcohol exposure during the early stages of embryo development directly affects epigenetic reprogramming and permanently alters genome-wide DNA methylation.

In this study, we used a pre-clinical mouse model of prenatal alcohol exposure to specifically target preimplantation embryos that are undergoing their epigenetic reprogramming wave. We show that this exposure leads to a surge in morphological brain defects during fetal life, and that exposed embryos with no visible abnormalities or developmental delays present lasting DNA methylation alterations in forebrain tissues, including sex-specific disparities in DNA methylation dysregulation.

\section{Results}

\section{Modeling early pre-implantation alcohol exposure} increases phenotypic alterations in developing embryos

To define the developmental and epigenetic (i.e., DNA methylation) impact of a binge alcohol exposure episode on early embryos undergoing the epigenetic reprogramming wave, we first established a pre-clinical mouse model to specifically expose pre-implantation embryos (E2.5) to short, but elevated alcohol levels. To avoid possible confounding effects of gavage-associated stress, we used a well-recognized two-injection paradigm [42-45]. Pregnant mice (C57BL/6) were subcutaneously injected at E2.5 (8-cell embryos) with two doses of $2.5 \mathrm{~g} / \mathrm{kg}$ ethanol (EtOH-exposed), or $0.15 \mathrm{M}$ saline (control), at $2 \mathrm{~h}$ intervals (Fig. 1a). Pregnant females reached a peak blood alcohol concentration (BAC) of $284.27 \mathrm{mg} / \mathrm{dL}$ (3 h) with an average of $158.31 \mathrm{mg} / \mathrm{dL}$ over a $4 \mathrm{~h}$ window (Fig. 1b). In contrast with other chronic prenatal alcohol exposure models [46-49], this short but acute level of ethanol exposure on pre-implantation embryos did not affect average mid-gestational (E10.5) litter size (Ctrl; $n=8.13 \pm 2.58$ vs EtOH-exposed; $n=7.91 \pm 2.86$; Fig. 1 c), or sex distribution (Ctrl and EtOH-exposed; $49 \%$ female vs $51 \%$ males; Fig. 1d). Similarly, early pre-implantation embryos subjected to binge-like alcohol levels did not show differences in mean morphological measurements at E10.5, however, we observed a significant increase in embryo-to-embryo variability for crown to rump length $(p<0.0001)$, head height $(p<0.01)$, occipital to nose diameter $(p<0.001)$ and brain sagittal length $(p<0.05)$ for EtOH-exposed embryos compared to controls (Fig. 1e). The greater intra-subject variability observed suggests that binge alcohol exposure levels during pre-implantation can alter the normal developmental programming of early embryos, thus causing abnormal morphological outcomes.

To define how a binge alcohol exposure episode during early pre-implantation can affect fetal development, we next investigated the morphological outcome of 


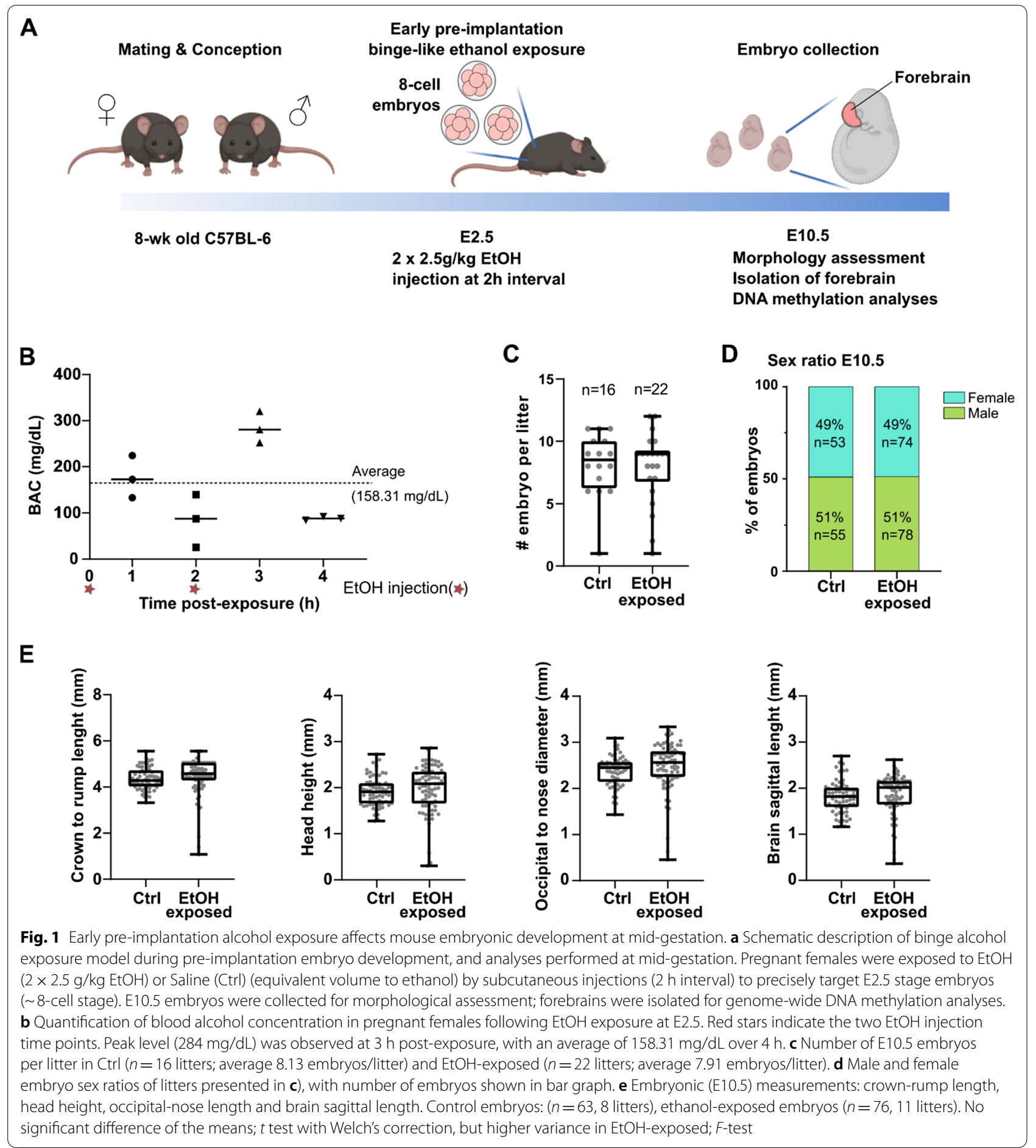

E10.5 embryos (control embryos: $n=108$ from 16 litters, EtOH-exposed: $n=152$ from 22 litters). As observed in Fig. 2a, early pre-implantation $\mathrm{EtOH}$ exposure leads to a significant increase in morphological defects or delayed development of mid-gestation embryos (19\% vs $2 \%$, $p<0.0001)$. Types of defects observed in EtOH-exposed embryos included brain anomalies (e.g., forebrain or midbrain malformations) (10\%), growth restriction or delayed development (5\%), heart defects (2\%), and other abnormal features (2\%) (Fig. 2b, c). There was no sexspecific phenotypic divergence between EtOH-exposed male and female embryos for morphological defects 


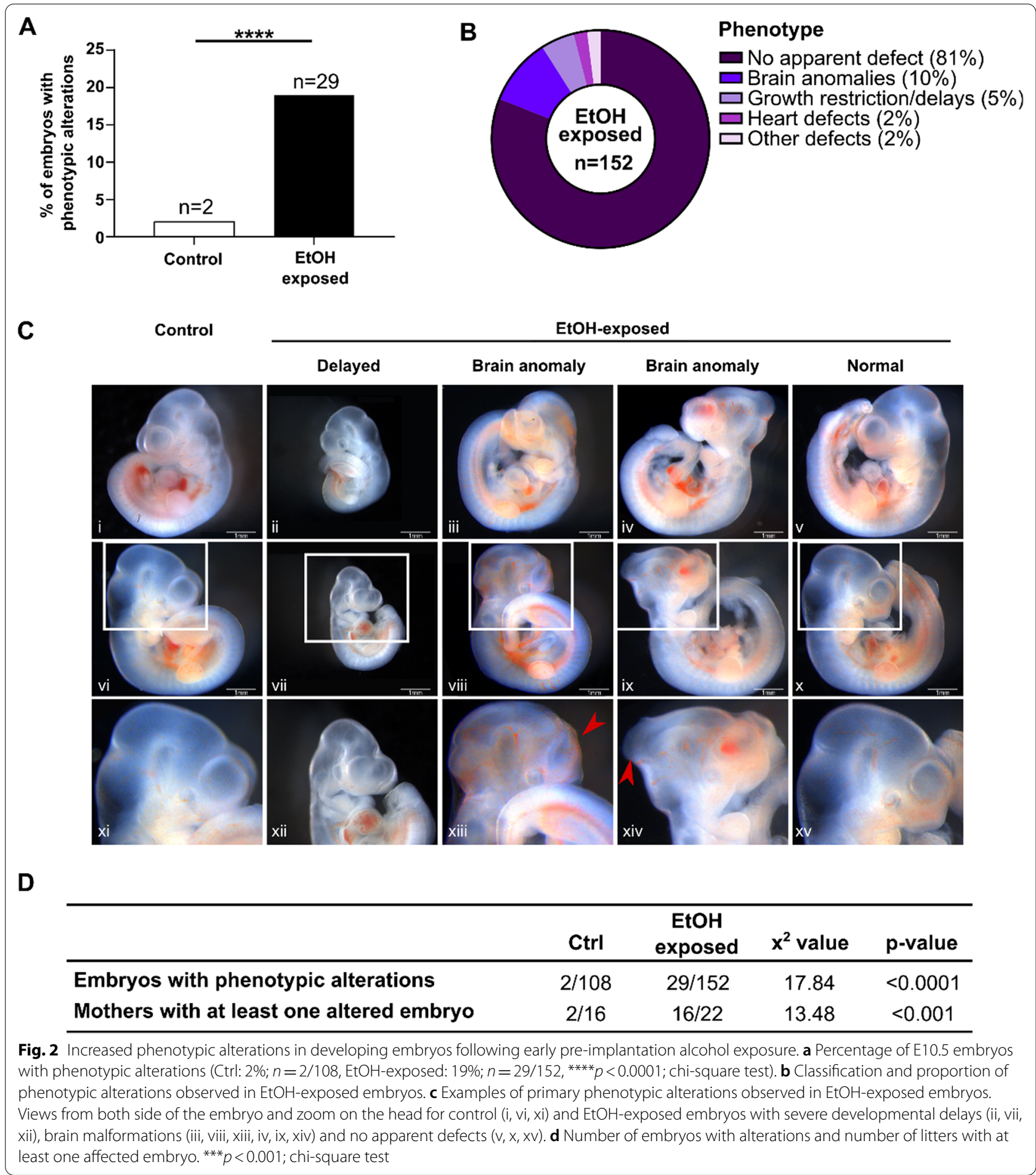

( $18 \%$ vs $20 \%$, Additional file 1: Fig. S1) or defect categories (Additional file 1: Fig. S1). Compared to controls, we observed that binge alcohol exposure during pre-implantation leads to a larger proportion of E10.5 embryos with phenotypic alterations $(29 / 152$ vs $2 / 108, p<0.0001)$, and that embryos with phenotypic alterations are distributed across most ethanol-exposed litters (16/22 vs $2 / 16$, $p<0.001$ ) (Fig. 2D) and not restricted to a small number of litters. Taken together, these results show that a binge alcohol exposure episode on pre-implantation embryos 


\begin{abstract}
(See figure on next page.)
Fig. 3 Early pre-implantation alcohol exposure triggers DNA methylation alterations in developing embryonic forebrain. Genome-wide CpG methylation analyses of E10.5 control $(n=6)$ and EtOH-exposed $(n=16)$ forebrains. a Distribution of genomic tiles (100 bp) (all-tiles; $n=114911)$ across ranges of $\mathrm{CpG}$ methylation levels in control and EtOH-exposed samples. ${ }^{* * *} p<0.0001,{ }^{* * *} p<0.001,{ }^{*} p<0.05$; $z$-test proportion test. $\mathbf{b}$ Heatmap showing CpG methylation levels for the top $1 \%$ most variable tiles $(n=1200)$ between control and EtOH-exposed forebrains. Gray lines in heatmap have no associated methylation values because of lack of sufficient sequencing coverage in sample. c Scatterplot representing the differentially methylated regions (DMRs) between control and EtOH-exposed forebrains (see "Methods" section for details). Red dots represent the tiles with a methylation increase of at least $10 \%$ in $\mathrm{EtOH}$-exposed compared to control forebrains $(n=69)$; blue dots represent the tiles with a methylation decrease of at least $10 \%$ in EtOH-exposed compared to control forebrains ( $n=1440)$; grey dots represent the tiles with changes less than $10 \%$ in EtOH-exposed compared to control forebrains $(n=113$ 402). d Proportion of DMRs associated with the changes of CpG methylation levels between control and EtOH-exposed E10.5 forebrains. e Comparison of CpG methylation levels of specific DMRs-associated tiles in EtOH-exposed versus control forebrains. Blue bar graph represents the comparison for decreased-methylation DMRs $(n=1440)$; red bar graph represents comparison for the increased-methylation DMRs $(n=69)$. f Functional enrichment analysis showing top 10 enriched pathways for decreased- and increased-methylation DMRs located in genic regions ( $n=710$ unique gene DMRs), based on Metascape analysis for pathways and $p$ value. The size of the dot represents the number of DMR-associated genes in a pathway, and gene ratio represents the number of DMR-associated genes with regards to the number of genes in a pathway. $\mathbf{g}$ Examples of $\mathrm{CpG}$ methylation levels of individual samples for DMR-associated genes related to the top enriched pathways. Letters under gene name relate to the pathways in $\mathbf{f}$ ). $¥$ represents significant differences in CpG methylation levels of DMRs (e.g., $\pm>10 \%$ methylation difference, $q<0.01$ ) between control and EtOH-exposed embryos (see "Methods " section for details)
\end{abstract}

undergoing the epigenetic reprogramming wave does not interfere with normal processes of implantation but leads to heterogeneity in morphological presentation during fetal life that mirrors the spectrum of clinical features associated to FASD.

\section{Pre-implantation alcohol exposure causes alterations in forebrain DNA methylation profiles}

To assess whether alcohol exposure during the embryonic epigenetic reprogramming wave dysregulates the normal programming of brain DNA methylation patterns during fetal development, we established genomewide DNA methylation profiles using rRRBS on E10.5 mouse forebrains. To mirror the $90 \%$ of children with FASD that show no dysmorphic features but still suffer from mild to severe neurological disabilities (e.g., learning deficits, intellectual delays), we randomly selected 6 controls (3 males; 3 females) and 16 ethanol-exposed embryos ( 8 males; 8 females) of similar size (i.e., embryo size, head height, occipital to nose diameter, brain sagittal length) with no visible morphological defects (Additional file 1: Fig. S2). Histological analysis revealed that EtOH-exposed embryos with no visible morphological defects or developmental delays had a general layout and distribution of brain cells that were comparable to controls (Additional file 1: Fig. S3A-B), whereas EtOH-exposed embryo with delayed development were distinctly different (Additional file 1: Fig. S3C). Furthermore, using markers for proliferation (Ki67 antigen) and apoptosis (cleaved Caspase-3), we confirmed that the early ethanol exposure did not promote an imbalance in cell proliferative response (Additional file 1: Fig. S4) or cell death (Additional file 1: Fig. S5) across brain regions of embryos with no apparent morphological defects. By removing embryos with abnormalities, developmental delays, or gross brain structure aberration, we further reduced potential DNA methylation variability that could be due to divergent forebrain cell type proportions between samples.

The first set of analyses was designed to define whether early pre-implantation alcohol exposure caused lasting DNA methylation alterations in developing E10.5 embryonic forebrains despite the absence of phenotypic presentation. To do so, we compared the average DNA methylation levels in $100 \mathrm{bp}$ non-overlapping genomic windows (tiles; see "Methods" section) between controls and $\mathrm{EtOH}$-exposed samples. After removal of sex chromosomes, we identified 114911 unique sequenced tiles containing 794803 common CpGs across samples (min. 5 samples/condition, $\geq 10 \times$ sequencing depth). When tiles were classified according to their DNA methylation levels, we observed significant changes in the distribution of categories, including tiles ranging between 90 and $100 \%$ methylation for which we observed a decrease in $\mathrm{EtOH}$-exposed forebrains compared to controls $(26 \%$ vs $24 \%, p<0.0001$ (Fig. 3a). Clustering of individual E10.5 forebrain samples by DNA methylation levels for the top $1 \%$ most variable tiles $(n=1200)$ revealed three main subgroups; a first essentially composed of control samples (6/9; right in heatmap), a second mainly composed of EtOH-exposed female samples with similarities to controls patterns (5/6; middle in heatmap), and a third mostly composed of male EtOH-exposed samples with highly divergent patterns (6/7; left in heatmap) (Fig. 3b). We next identified regions of the genome that showed altered DNA methylation levels $( \pm \geq 10 \%$ mean differences between tiles; see "Methods") as a result of the early pre-implantation alcohol exposure. Using such criteria, we identified 1509 differentially methylations regions (DMRs) with significant DNA methylation level 


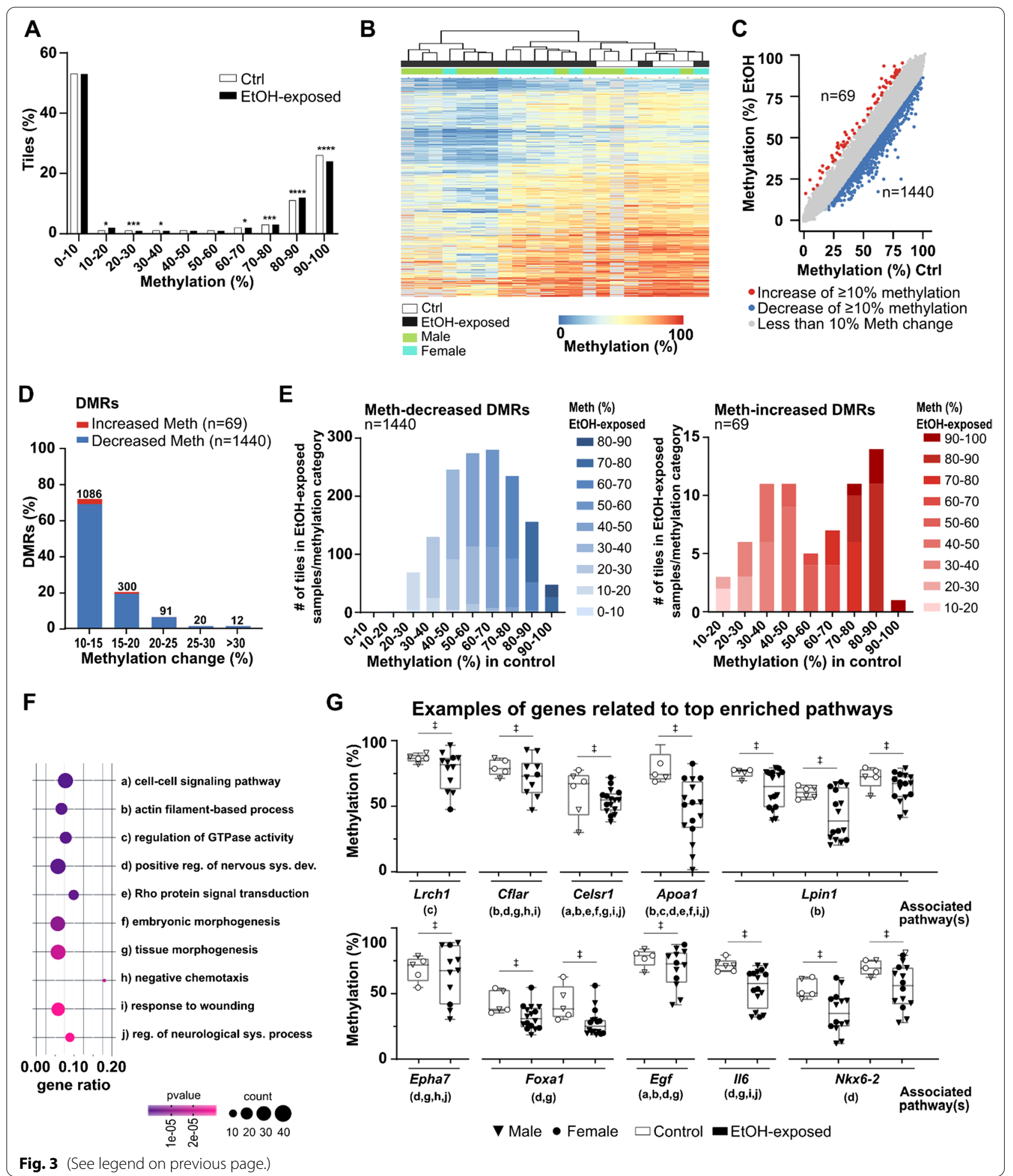

decrease $(n=1440)$ or increase $(n=69)$ in EtOH-exposed forebrains compared to control forebrains (Fig. 3c). Differences in methylation levels ranged from 10 to $55 \%$, with most DMRs showing $10-15 \%(n=1086 ; 72 \%)$ or
$15-20 \%(n=300 ; 20 \%)$ methylation change between EtOH-exposed and control forebrains (Fig. 3d). Most DMR-associated tiles with decreased methylation levels in $\mathrm{EtOH}$-exposed samples $(n=1239)$ were highly 
(See figure on next page.)

Fig. 4 Sex-related changes in embryonic forebrain DNA methylation mediated by early pre-implantation ethanol exposure. a Schematic design of sex-related genome-wide $\mathrm{CpG}$ methylation analysis in male $(\mathrm{Ctrl} n=3, \mathrm{EtOH} n=8)$ and female $(\mathrm{Ctrl} n=3, \mathrm{EtOH} n=8)$ E10.5 forebrain samples. Identification of all-tiles associated with either male samples $(n=83424)$, female samples $(n=126857)$, male-female common samples $(n=46$ 475), as well as male-specific $(n=971)$, female-specific $(n=133)$ and common $(n=111)$ DMRs (see "Methods" section for details). b Distribution of genomic tiles (100 bp) (common tiles; $n=46475$ ) across ranges (10\%) of CpG methylation in male and female control and EtOH-exposed samples ${ }_{* * * *} p<0.0001,{ }^{* *} p<0.01,{ }^{*} p<0.05$; $z$-test proportion test. $\mathbf{c}$ Distribution of common DMRs $(n=111)$ with increased or decreased CpG methylation in male (outer circle) and female (inner circle) samples. $\mathbf{d}$ Proportion of common DMRs associated with the changes of CpG methylation levels between control and EtOH-exposed in male and female forebrains. e Percentage of tiles associated with various genomic features: genomic annotation (left), repeat elements (middle) and CpG-rich context (right) in common all-tiles ( $n=46475)$ and common DMRs $(n=111)$. $\mathbf{f}$ Examples of CpG methylation levels of individual samples of common DMRs in male and female samples. $¥$ represents significant differences in CpG methylation levels of DMRs (e.g., $\pm>10 \%$ methylation difference, $q<0.01$ ) between control and EtOH-exposed embryos (see "Methods" section for details)

methylated $(\geq 40-50 \%)$ in control samples, whereas DMRs that gained methylation in EtOH-exposed samples had variable levels in controls (Fig. 3e). The DMRs mainly overlapped with intergenic (43\%) and genic (53\%: introns; $37 \%$, exons; $12 \%$, and promoters; $4 \%$ ) regions. Gene ontology enrichment analyses associated genic DMRs with various processes, including cell-to-cell signaling pathway, regulation of GTPase activity, positive regulation of nervous system development, tissue and embryonic morphogenesis, as well as regulation of neurological systems (Fig. 3f). When we looked at the DNA methylation levels between control and $\mathrm{EtOH}$-exposed forebrains for genes associated to these enriched pathways (e.g, Lrch1, Cflar, Celsr1, Apoa1, Lpin1, Epha7, Foxa1, Egf, Il6, Nkx6-2), we observed increased inter-individual variability in methylation levels for $\mathrm{EtOH}$-exposed forebrains with some samples or genomic regions being more affected than others (Fig. 3g).

Overall, we showed that alcohol exposure during the embryonic epigenetic reprogramming wave triggers an array of DNA methylation alterations observed in forebrain of embryos that presented no visible abnormalities or developmental delays at E10.5.

\section{Sex-specific DNA methylation alterations following pre-implantation alcohol exposure}

We next sought to discern whether early pre-implantation alcohol exposure could have a sex-specific impact on later forebrain DNA methylation patterns. By analyzing male and female samples separately (min. 3 samples/ condition/sex, $\geq 10 \times$ sequencing depth), we identified 83424 and 126857 unique sequenced tiles in male and female forebrains, respectively (Fig. 4a). Although there were lesser regions analyzed in males, they showed a larger number of DMRs following pre-implantation alcohol exposure (DMRs: males $n=2097$, females $n=1273$ ) (Additional file 1: Fig. S6). In male EtOH-exposed forebrains, we found 2097 DMRs of which 1936 (92\%) showed decreased methylation. Comparably, female EtOH-exposed forebrains revealed 1273 DMRs of which
1066 (84\%) presented decreased methylation (Additional file 1: Fig. S6A-D). We also observed contrasts between DMR number, as well as associated biological processes among sexes (Additional file 1: Fig. S6E). However, we do not exclude that these results could be related to only a partial overlap in sequenced regions $(n=46475)$ between male and female forebrain samples (Fig. 4a).

To circumvent this issue, we focused on the 46475 regions with sufficient sequencing coverage in both sexes encompassing 373530 CpGs (Fig. 4a). We did not observe any differences in global DNA methylation levels between sexes in either controls or EtOH-exposed forebrains (males: $29.4 \%$ vs $28.7 \%$, females: $29.4 \%$ vs 29.2\%; not shown). However, when these common tiles ( $n=46475)$ were distributed according to their DNA methylation levels in control and $\mathrm{EtOH}$-exposed forebrains, we observed a greater shift in tile distribution in male EtOH-exposed samples (Fig. 4b), suggesting a greater effect on males. Accordingly, among the 46475 male-female common tiles, we identified 971 male-specific DMRs, 133 female-specific DMRs, and 111 DMRs that were present in both sexes (Fig. 4a). Out of these 111 common DMRs, 100 had similar alteration profiles (i.e., $n=99$ DMRs with decreased methylation, $n=1$ DMR with increased methylation) in male and female EtOHexposed forebrains (Fig. 4c). For the other DMRs $(n=11)$, the alcohol exposure caused conflicting alteration profiles between sexes (e.g., decreased-methylation in males vs increased-methylation in females). For the most part, the common DMRs displayed low levels of methylation changes (10-15\% range) for both sexes (42\% for males, $79 \%$ for females), with methylation changes greater than $25 \%$ only present in male EtOH-exposed samples (Fig. 4d). When compared to the 46475 common tiles analyzed, the 111 common DMRs were more enriched in intergenic regions and intron categories, as well as for LINE elements, whereas they were mostly depleted of $\mathrm{CpG}$ rich sequences (i.e. CpG islands). In addition, common DMRs in male and female EtOH-exposed forebrains showed various levels of DNA methylation for 


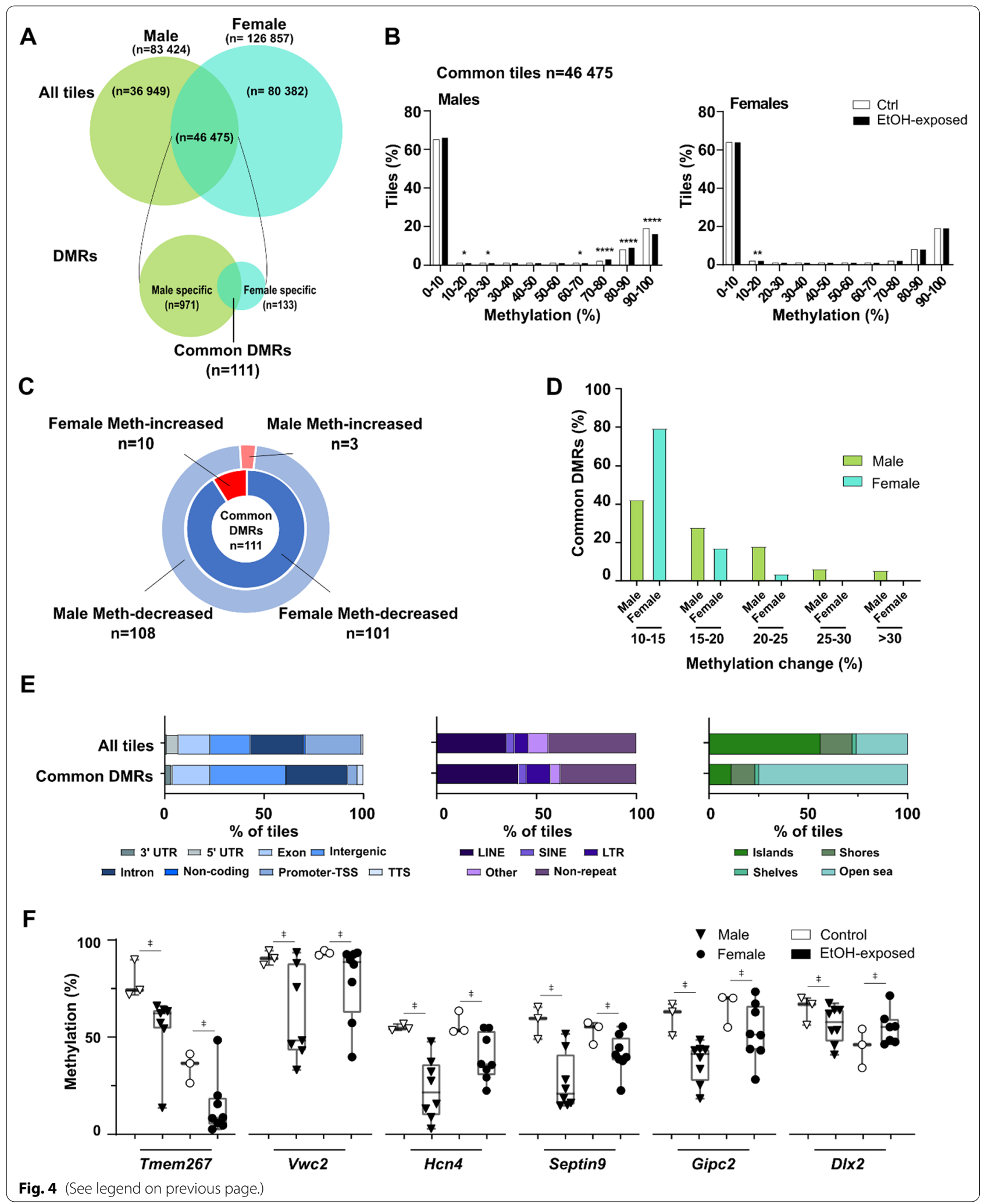




\begin{abstract}
(See figure on next page.)
Fig. 5 Early pre-implantation ethanol exposure induces sex-specific DNA methylation alterations in developing embryonic forebrain. Identification of male and female sex-specific DMRs in EtOH-exposed E10.5 forebrains is described in Fig. 4a. a Manhattan plot showing differences of CpG methylation by chromosomal position for male-specific ( $n=971$; left graph) and female-specific ( $n=133$; right graph) DMRs. Red dots represent DMRs with increased-methylation in EtOH-exposed forebrains ( $n=31$; right graph and $n=30$; left graph); blue dots represent DMRs with decreased methylation in $\mathrm{EtOH}$-exposed forebrains ( $n=940$; right graph and $n=103$; left graph); grey dots represent tiles with less than $10 \%$ methylation differences between EtOH-exposed and control forebrains ( $n=45$ 504; right graph and $n=46$ 342; left graph). b Proportion of sex-specific DMRs (upper graph; male $n=971$, lower graph; female $n=133$ ) associated with changes in $\mathrm{CpG}$ methylation levels between control and EtOH-exposed E10.5 forebrains. c Percentage of sex-specific DMR-associated tiles (male $n=971$, female $n=133$ ) across various genomic features: genomic annotation (top), repeat elements (middle) and CpG-rich context (bottom). $\mathbf{d}$ Percentage of CpG methylation levels of male- and female-specific DMRs based on the distribution of CpG sites in CpG islands, CpG shores and CpG shelves. e Functional enrichment analysis showing top enriched pathways for male-(top 10 pathways, $n=508$ unique gene DMRs) and female-specific DMRs ( $n=87$ unique gene DMRs), based on Metascape analysis for pathways and $p$ value. The size of the dot represents the number of DMR-associated genes in pathways, and gene ratio represents the number of DMR-associated genes with regards to the number of genes in a pathway. $\mathbf{f}$ Examples of CpG methylation levels of individual samples for sex-specific DMR-associated genes related to the top enriched pathways in $\mathbf{e}$ ). Letters under gene name relate to the pathways in $\mathbf{e}$ ). $¥$ represents significant differences in CpG methylation levels of DMRs (e.g., $\pm>10 \%$ methylation difference, $q<0.01$ ) between control and EtOH-exposed embryos (see methods section for details)
\end{abstract}

an assortment of genes, including Tmem267 (putative oncogene), $V w c 2$ (neural development and function), Hcn4 (cardiac function), Septin9 (cytoskeletal formation), Gipc2 (gastrointestinal processes) and $D l x 2$ (forebrain and craniofacial development) (Fig. 4f, Additional file 1: Fig. S7).

When we turned our attention to male-specific $(n=971)$ and female-specific $(n=133)$ DMRs within the 46475 common tiles, we observed that pre-implantation alcohol exposure had a more profound impact on male forebrains in terms of DMR number and level of methylation changes (Figs. 4a, 5a). Although a portion of observed DMRs showed increased methylation (males 3\%; $n=31$, females 23\%; $n=30$ ), the majority of sex-specific DMRs were associated with a partial loss of DNA methylation (males $n=940$, females $n=103$ ) in all chromosomes. The only chromosomes that showed an enrichment $(p<0.0001)$ in DMRs were female X-chromosomes $(n=48 / 133)$. Most of these sex-specific DMRs showed low levels of alterations, in the $10-15 \%$ range (males $n=627$ DMRs; 65\%, females $n=112$ DMRs; $84 \%$ ), with males having a larger proportion of DMRs with $>15 \%$ methylation changes (Fig. 5b). When we highlighted genomic features associated to these sex-specific DMRs, we observed more female-specific DMRs in promoter regions (17\% vs $4 \%$ ) and CpG rich regions (33\% vs $8 \%$ ) when compared to male-specific DMRs (Fig. 5c). When we focused on the distribution of $\mathrm{CpG}$ sites in a sequence context (i.e., CpG islands, shores, shelves), we noticed a significant loss of global methylation in malespecific DMRs for male EtOH-exposed forebrains, and similarly for female-specific in female EtOH-exposed forebrains (Fig. 5d). For female-specific DMRs, we noticed that DNA methylation levels in control forebrains are all higher in females compared to males. These higher methylation levels are mainly associated with the presence of methylation marks associated to the X-inactivation process in females (female-specific DMRs on X-chromosome: CpG islands 35/44; shores 9/36, shelves $0 / 6$. Figure $5 \mathrm{f}$ bottom for examples). The sex-specific DMRs resulting from pre-implantation alcohol exposure were related to divergent biological processes between males (e.g., muscle differentiation, cell projection organization) and females (e.g., receptor protein tyrosine kinase pathway, epithelial cell migration) (Fig. 5e, f).

We then evaluated whether early embryonic alcohol exposure led to expression errors in the forebrains of E10.5 embryos by focussing on a group of genes (with or without DMRs) implicated in the regulatory network coordinating the timing of GABAergic interneuron migration and forebrain formation. At the core of this network is the $D l x$ family of homeodomain transcription factors $[50,51]$. We show that Dlx2 (DMR in gene body; males and females) and $D l x 1$ (no DMRs) have small but significant changes in gene expression (Dlx2: males and females; $D l \times 1$ : females) (Additional file 1: Fig. S8A), whereas for $D l x 5$ and $D l x 6$ (no DMRs), the expression remained unchanged (Additional file 1: Fig. S8B). Upstream key regulator of MGE (medial ganglionic eminence)-derived GABAergic interneurons [52-54], $N k x 2.1$ (no DMRs) did not show expression alterations (Additional file 1: Fig. S8B), however downstream transcription factors such as Sox6 (DMR in gene body; males), and Arx (direct target of $\operatorname{Dlx} 2[55,56]$ ), DMR in promoter; females) showed small but significant gene expression alteration (Sox6; males and females, Arx; males) (Additional file 1: Fig. S8A).

Together, these results indicate that alcohol exposure in pre-implantation embryos in conjunction with epigenetic reprogramming leads to variable levels of sex-specific alterations, with male embryonic forebrains being more prone to DNA methylation alterations. This suggests that 


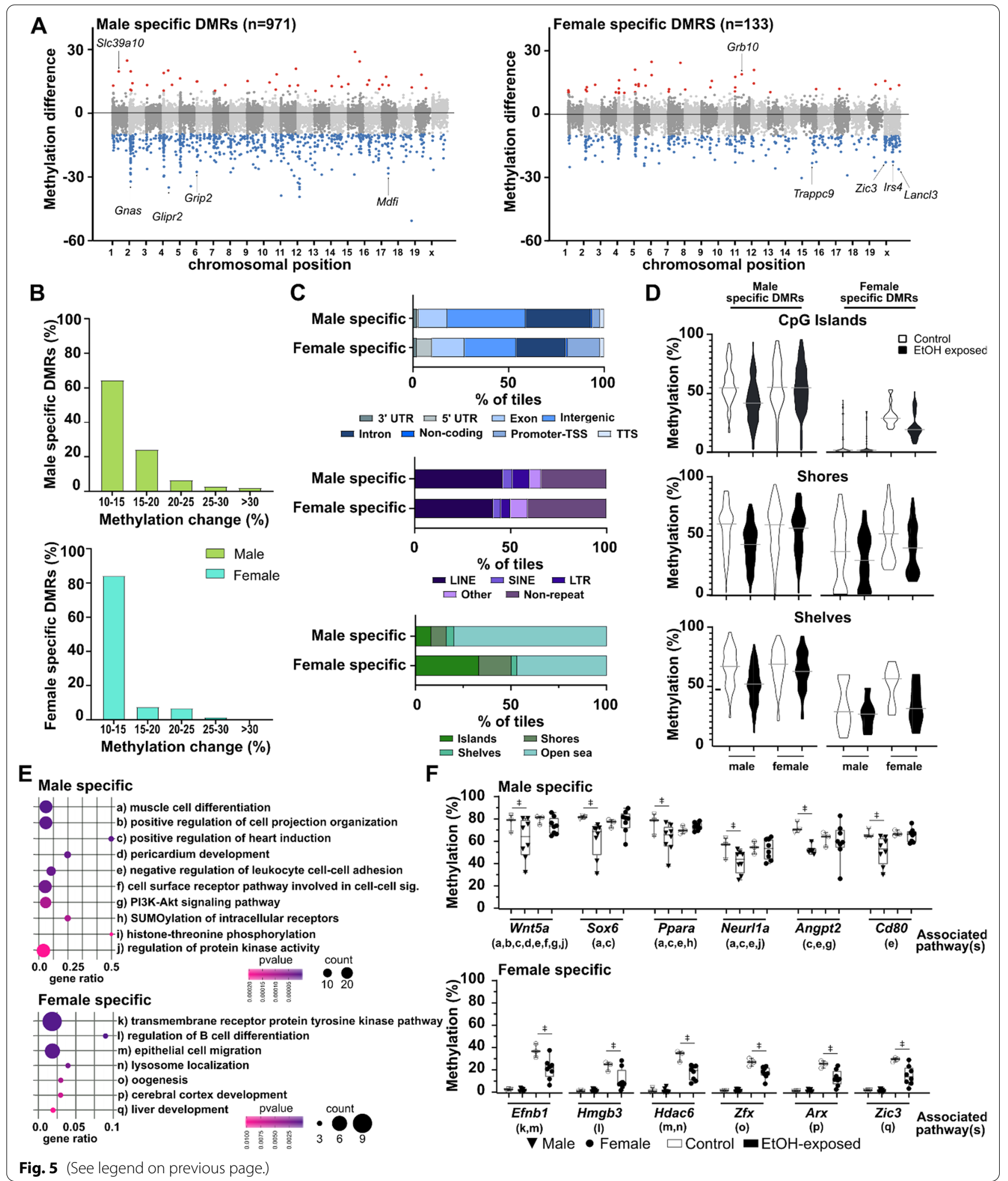

pre-implantation male embryos are more susceptible to the initial adverse exposure rendering them less efficient at re-establishing proper DNA methylation patterns during the de novo methylation wave, or that female embryonic cells are better at rectifying dysregulated DNA methylation patterns during development. 


\section{Pre-implantation alcohol exposure leads to partial loss} of imprinted DNA methylation patterns

To further determine if a binge alcohol exposure episode on early embryos undergoing the epigenetic reprogramming wave is more adverse on the DNA methylation patterns of male or female embryos, we directed our attention to ICRs of imprinted genes, which are well known for their key roles in brain development and growth. We and others have shown that allele-specific methylation maintenance is required on ICRs during the reprogramming wave, as partial or complete loss of ICR profiles is permanent. Since our alcohol exposure specifically targets E2.5 embryos (8-cell stage), dysregulation in ICRs methylation maintenance would still be detectable in E10.5 forebrains. From the 46475 commonly sequenced tiles, 28 were located within the ICRs of 9 imprinted genes. Our non-allele specific differential methylation analysis revealed that out of these 28 regions, 24 were differentially methylated in male $\mathrm{EtOH}$-exposed forebrains. These 24 DMRs were associated to imprinted genes H13, Nnat, Gnas, Kcnq1, Plagl1, Zrsr1, Peg13 and Igf $2 r$ (Fig. 6a). In females, only 2 of those 28 tiles showed altered levels in female EtOH-exposed forebrains, which were associated with Gnas and Grb10. We expanded our search for dysregulated ICR DNA methylation patterns within uniquely sequenced regions in male $(n=36949$ tiles) and female ( $n=80382$ tiles) forebrains (Fig. 4a) to retrieve additional ICR-associated tiles. Again, in EtOHexposed male forebrains, the majority of ICRs (10/13 tiles) showed altered DNA methylation patterns (Fig. 6b), which were associated with Gnas, Snrpn, Peg3, Plagl1, Zrsr1 and Impact. Conversely, in EtOH-exposed female forebrains, we now observed a large portion of ICRs (19/36 tiles) with dysregulated DNA methylation levels associated to various imprinted genes (i.e., Gnas, Peg10, Inpp5f, Kcnq1, Snrpn, Grb10, Zrsr1, Peg13, Slc38a4, Igf2r and Impact). When we plotted individual sample DNA methylation values for these ICR-associated DMRs, we again observed a high degree of heterogeneity in $\mathrm{EtOH}-$ exposed forebrains with some samples showing altered DNA methylation levels (e.g., partial loss, complete loss), and others revealing normal control methylation values (Fig. 6a-c).

This partial loss of DNA methylation signatures within ICRs of male and female E10.5 EtOH-exposed forebrains was associated with small but significant deviation in expression level for a number of imprinted genes (e.g., Gnas, Plagl1, Peg13) (Additional file 1: Fig. S9A). Whereas for others, although we observed similar ICRassociated DMRs, we did not detect any alteration in imprinted gene expression (e.g., Impact, Peg10, Grb10) for either male or female E10.5 EtOH-exposed forebrains (Additional file 1: Fig. S9B).
Both male and female EtOH-exposed forebrains showed variable levels of DNA methylation alterations in imprinted gene ICRs, which suggests that a bingelike alcohol exposure during pre-implantation interferes with the DNA methylation maintenance machinery during the epigenetic reprogramming wave. Finally, these results also suggest that male pre-implantation embryos are more susceptible to the initial adverse of alcohol exposure.

\section{Discussion}

In this study, we showed that a binge alcohol exposure episode on early-stage embryos (8-cell; E2.5) leads to a surge in morphological brain defects and delayed development during fetal life, that are reminiscent of clinical features associated to FASD. As seen in children exposed to alcohol prenatally, a portion of ethanol-exposed embryos presented a spectrum of alcohol-induced macroscopic defects while the majority showed no noticeable dysmorphic features and no alterations. However, forebrain tissues from ethanol-exposed embryos with no visible macroscopic abnormalities, developmental delays, alteration in cell proliferative response or cell death still presented lasting genome-wide DNA methylation alterations in genes associated to various biological pathways, including neural/brain development, and tissue and embryonic morphogenesis. These ethanol-exposed embryos also showed partial loss of imprinted DNA methylation patterns for various imprinted genes critical for fetal growth, development, and brain function. Moreover, we observed alcohol-induced sex-specific errors in DNA methylation patterns with male embryos showing increased vulnerability.

\section{Modeling early pre-implantation alcohol exposure}

One of the challenges when modeling FASD is unscrambling direct and indirect outcomes associated with the amount, pattern (continuous vs. binge drinking), and developmental timing of alcohol exposure [18, 19, $45,57-63]$. By targeting pre-implantation embryo, we observed that a binge-like alcohol exposure on a single embryonic cell type (8-cell stage blastomeres) leads to increased rate of macroscopic defects (e.g., brain anomalies, growth restriction, heart defects) across litters, with no impact on litter size or on sex-specific phenotypic representation during fetal life. These findings corroborate with pioneer work that reported abnormal fetal development without significant reduction in litter size following exposure during pre-implantation $[64,65]$. However, the acute dosage regimen paradigms used in those studies resulted in a much higher rate $(67-100 \%)$ of embryonic abnormalities and severe growth retardation, as well as fetal death $[41,66]$. Nevertheless, such 


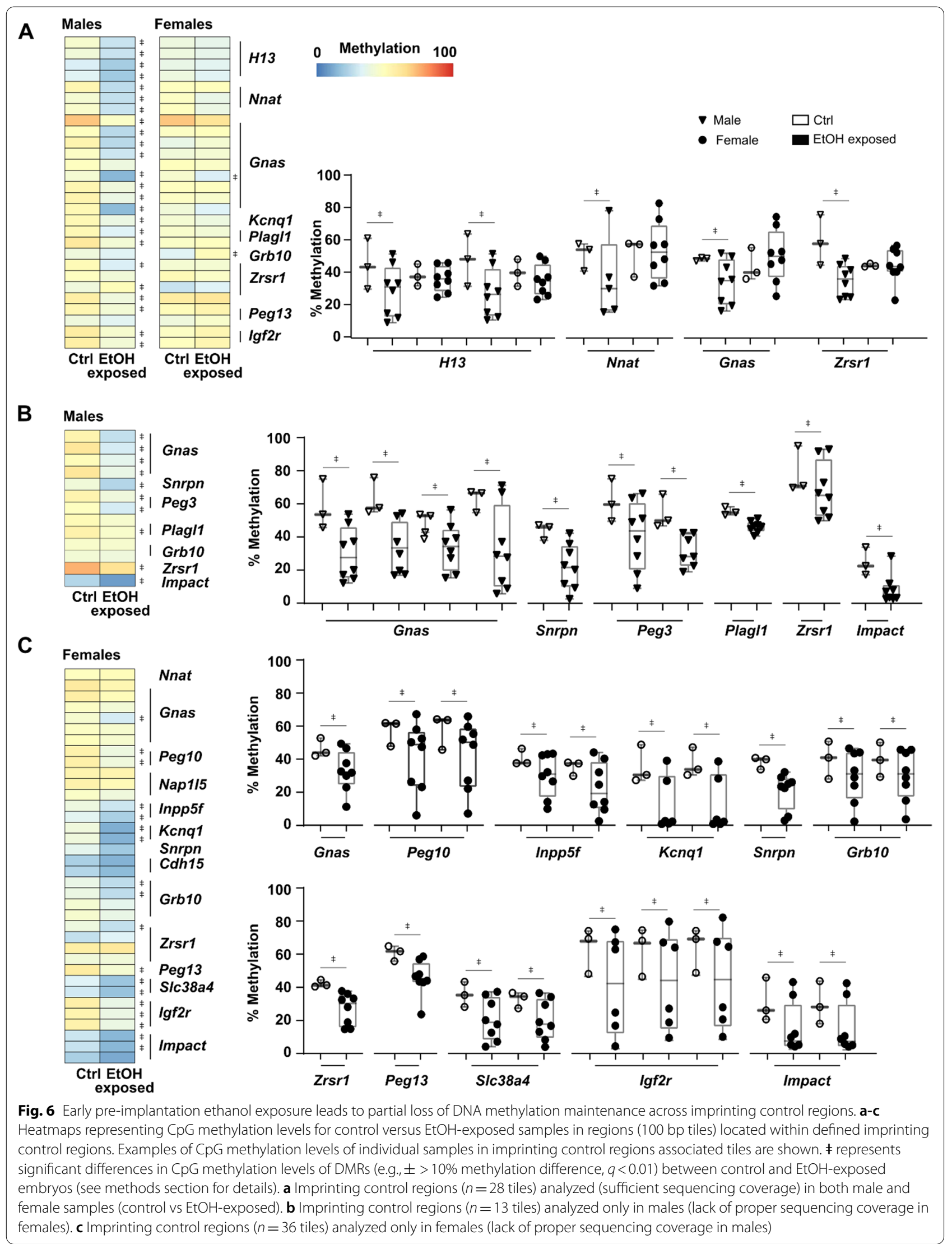


studies along with others [67] confirm that even prior to direct maternal-fetal interface exchanges via the placenta, alcohol can reach the developing pre-implantation embryos through the female reproductive track. In vitro studies support that pre-implantation embryos are sensitive and negatively affected by alcohol exposure [68]. Although less investigated and understood than maternal exposure, studies suggest that alterations (e.g., epigenetic errors) initiated on the fathers' sperm are passed-on during fertilization to pre-implantation embryos, influence development beyond implantation, and lead to abnormal offspring development (e.g., fetal growth restriction, birth defects, placental defects) [58, 69-72].

It remains to be defined whether some of the milder abnormalities or delays that we observed at mid-gestation would become resolved or accentuated by birth, and whether embryos that presented no visible abnormalities or developmental delays but had DNA methylation alterations would show cognitive dysfunctions as observed in other FASD models and children with FASD. In parallel, outlining if the dysregulation in DNA methylation profiles is associated with abnormal migration and organization of specific brain cell subtypes during development would further extend our understanding about the effect of early pre-implantation alcohol exposure on the neurobiological phenotype in offspring.

\section{Pre-implantation alcohol exposure leads to partial loss of imprinted DNA methylation}

Our genome-wide, high-resolution analysis of ethanolinduced DNA methylation alterations highlighted partial loss of DNA methylation maintenance within various $100 \mathrm{bp}$ tiles located across imprinting control regions (e.g., Gnas, Zrsr1, Impact) in E10.5 male and female forebrains. Then again, methylation alterations were not necessarily widespread across entire imprinting control regions, or in all individual ethanol-exposed embryos, suggesting only a slight reduction in DNMT1/DNMT1o maintenance activity. Concordantly, some imprinted genes showed alterations in their expression profiles (e.g., Gnas, Plagl1, Peg13) in both male and female ethanol-exposed forebrains, whereases others (e.g., Impact, Peg10, Grb10) were not affected. We know that temporary lack of DNA methylation maintenance by DNMT1o in 8-cell embryos (E2.5) leads to delays in development and to a wide range of lethal anatomical abnormalities that are associated with epigenetically mosaic embryos that failed to properly maintain normal imprinted methylation patterns at mid-gestation [73-75]. In zebrafish, alcohol exposure during the two first days of embryo development led to reduced levels of Dnmt1 expression [76], which could ultimately lead to a temporary reduction in methylation maintenance activity. Studies aiming at defining how pre-implantation embryos respond to environmental stimuli (e.g., assisted reproductive technologies, toxicants, ethanol) have broadly explored the impact on imprinted genes, but have mainly relied on evaluating DNA methylation levels of imprinting control regions using targeted approaches (i.e., specific genomic loci) [41, 77, 78]. Although informative, conclusions are often based on profiling DNA methylation levels of a limited number of CpG sites associated to a handful of genes. For instance, modeling binge alcohol exposure during two consecutive days (E1.5, E2.5) led to decreased fetal and placental weight by E10.5, but only to a partial loss of DNA methylation in the H19-a negative regulator of growth and proliferation-imprinting control region (only $17 \mathrm{CpG}$ analysed) in the placenta. Although only a portion of one ICR was evaluated, these results insinuated that embryonic imprinted methylation was not affected by such early pre-implantation alcohol exposure.

Since imprinted genes are well recognized in regulating essential neurodevelopmental processes, including neural differentiation, migration and cell survival [79], we can presume that the forebrain of ethanol-exposed embryos with severe macroscopic defects and developmental delays would have broader loss of imprinted DNA methylation patterns compared to those with no visible abnormalities. However, the fact that morphologically normal forebrain tissues derived from ethanol-exposed pre-implantation embryos reveal widespread low-level DNA methylation alterations with increased inter-individual variability in imprinted control regions supports that failure in maintaining accurate methylation imprints could contribute to the invisible nature of FASD.

\section{Pre-implantation alcohol exposure initiates sex-specific DNA methylation programming errors}

We demonstrated that exposing 8-cell embryos to alcohol is detrimental for future forebrain autosomal and X-chromosome DNA methylation patterns. Furthermore, such an early embryonic exposure leads to sex-specific DNA methylation alterations with male embryos being more susceptible to alterations. Early embryonic developmental stages are marked by a series of molecular events that are crucial for the proper establishment of the developmental program, as well as the de novo genome-wide DNA methylation signatures that are conserved throughout development $[29,80]$. A considerable amount of evidence shows that interfering with this process in vivo or in vitro leads to sex-specific long-term effects in the offspring [36, 72, 81-87]. Although the mechanism remains unclear, we know that molecular events in early-stage embryos are marked with sex-specific discrepancies. For instance, mammalian pre-implantation embryos display 
differential expression of sex chromosome and autosomal transcripts, leading to extensive transcriptional sexual dimorphism $[88,89]$. In mouse, sex-biased gene expression $(n=69$; mainly X-linked) is detected as early as the 8 -cell stage with substantial variation between individual embryos, and with female embryos expressing higher transcript levels. Then, just prior to the de novo re-methylation wave, the sex chromosomes seem to further drive this sexual dimorphism in transcriptional regulation for hundreds of autosomal genes, with Dnmt $3 a$ and Dnmt3b showing higher expression in male blastocyst [90]. This is consistent with sex-specific acquisition of DNA methylation reported in bovine blastocyst, with males showing increased levels $[89,91]$. Thus, the alcohol-induced sex-specific DNA methylation alterations resulting from an early embryonic alcohol exposure model are mostly initiated at the 8-cell stage because of the male-female differences in transcriptional regulation. The DNA methylation alterations would then trigger a series of events that would negatively impact the re-establishment of genome-wide de novo DNA methylation profiles occurring between E3.5 and E6.5 in a sex-specific manner, with males being more affected. Once established, these abnormal de novo DNA methylation profiles would be maintained or would initiate further DNA methylation alterations during subsequent developmental stages. Since the sex-specific alterations observed in the forebrains are linked to divergent biological pathways, this could lead to sex-specific neurocognitive impairments in offspring. Although such observations have been made in children with FASD, with males appearing to be more vulnerable to the irreversible effects of fetal alcohol exposure (e.g., cranial and facial malformations, learning disabilities, social and memory disabilities, and altered brain structure and function) (reviewed in [92]), further studies are needed to explore the long-term degree and impact of the sexual dimorphism in this pre-implantation alcohol exposure paradigm.

\section{Pre-implantation alcohol exposure and impact on neurodevelopment}

One common phenotype observed in human FASD and animal models of prenatal alcohol exposure is the interference of alcohol on the development of the central nervous system (structural or functional abnormalities). We observed DNA methylation alterations in promoters and bodies of genes implicated in brain and nervous system function and development (e.g., Dlx2, Epha7, Foxa1, Nkx6, Vwc2, Sox6). For example, $D l x 2$ (Distal-Less Homeobox 2), is part of a transcription factor family $(D l x 1 / 2 ; D l \times 3 / 4 ; D l \times 5 / 6)$ that is a critical molecular determinant for forebrain and craniofacial development, as well as for coordinating the timing of GABA(gamma-aminobutyric acid)ergic interneuron migration and process formation [93-96]. $D l x 2$ is required to promote the expression of several downstream factors including other $D l x$ genes and Arx (directly activated by $D l x 2$ ) [56], an X-linked gene that also controls cortical interneuron migration and differentiation [97], which incidentally showed altered DNA methylation in female ethanol-exposed forebrains. Ethanol-exposed forebrains showed reduced methylation in Dlx2 (gene body) and $A r x$ (promoter), which correlated with reduced $D l x 2$ and Arx expression, whereas the average expression of $D l x 5$ and $D l x 6$ (no DMRs) remained unchanged. Mice lacking $D l \times 1 / 2$ have profound deficits in tangential migration of GABAergic cortical interneurons and neurite growth. Similarly, prenatal stress-induced anxiety in mouse leads to GABAergic interneuron deficiency-associated dysregulation of promoter DNA methylation levels of Gad67 (glutamic acid decarboxylase 67), an enzyme critical for GABA synthesis (the principal inhibitory neurotransmitter). In prenatal alcohol exposure models targeting different brain developmental time points, the migration and positioning of GABAergic cortical interneurons are profoundly impaired, leading to subsequent cortical dysfunction [98102]. We know that cortical interneuron dysfunction, associated with impaired development, migration, or function of interneurons, results in interneuronopathies that contribute to multiple neurodevelopmental disorders including autism, epilepsy, schizophrenia, and FASD [103-105]. We also know that GABAergic interneurons are particularly responsive to adverse maternal exposures during in utero periods of developmental plasticity; from the moment these cells arise, to the shaping of cortical circuits $[101,105]$. Our data suggest that early embryonic alcohol exposure triggers alterations in the epigenome that leave lasting signals that could lead to pathological plasticity in the developing brain. We need to further define whether GABAergic interneurons, or other cortical neurons, are particularly vulnerable to early embryonic alcohol-induced epigenetic programming errors, and whether this could drive interneuronopathies or neurodevelopmental impairments associated with FASD.

\section{Study limitation}

One limitation to our study is the absence of information about the dose-dependency of alcohol exposure with regards to the phenotypes and molecular consequences observed. Since higher peak blood alcohol concentrations have been shown to play a critical role in the extent of prenatal alcohol exposure-related damages [17, 18, 45, $48,66,86,106]$, we can presume that similar observations would be observed using our paradigm. Nonetheless, the impact of low-dose and early prenatal alcohol exposure 
should not be overlooked, as they have been associated to increase the risk of mental illness regardless of a FAS or FASD diagnosis in human (review in [107]). To fully comprehend the wide range of detrimental consequences (visible and invisible) associated to dose-dependency of alcohol exposure during pre-implantation will require a thorough investigation across pre- and post-natal development.

\section{Conclusion}

We showed that pre-implantation alcohol exposure is detrimental for normal development and leads to a broad spectrum of adverse outcomes that closely replicate clinical facets observed in children with FASD. Specifically, we demonstrate that a binge-like drinking episode while pre-implantation embryos are in the mist of their reprogramming wave leads to two main categories of lasting programming errors: the partial loss of DNA methylation at several imprinted control regions, and the abnormal re-establishment of de novo DNA methylation profiles in key biological pathways (e.g., neural/brain development, tissue and embryonic morphogenesis). Further studies in peri-implantation embryos, when DNA methylation is globally reacquired, and in specific cell subtypes during key neocortex developmental time points will provide a better understanding of the fundamental mechanisms leading to these DNA methylation programming errors and their implication in neurodevelopmental FASD-related deficits. Importantly, our data demonstrate that pre-implantation alcohol exposure does not lead to an "all-or-nothing" response, as morphologically normal embryos still presented conserved and sex-specific DNA methylation alterations in forebrain tissues, which could be indicative of the particular sexual dimorphism in cognitive dysfunctions associated with FASD. Thus, our study provides strong scientific evidence to refute the "all-or-nothing" principle and supports the potential contribution of early embryonic epigenetic alterations to the manifestation of neurodevelopmental phenotypes observed in a portion of children with FASD.

\section{Methods}

\section{Pre-implantation embryo binge-like ethanol exposure model}

Animal work was approved by the Comité Institutionnel de Bonnes Pratiques Animales en Recherche (CIBPAR) of the CHU Ste-Justine Research Center under the guidance of the Canadian Council on Animal Care (CCAC). Female C57BL/6 mice (8-week-old) were mated with same age C57BL/6 males (Charles River laboratories). Females that showed copulatory plugs the next morning were considered pregnant with day 0.5 embryos (E0.5).
They were separated from the males and housed together in a $12 \mathrm{~h} \mathrm{light/dark} \mathrm{cycle} \mathrm{with} \mathrm{unlimited} \mathrm{access} \mathrm{to} \mathrm{food}$ and water.

Using a recognized prenatal binge-like alcohol exposure paradigm $[41,45,66,106]$, pregnant females (E2.5) were injected with 2 subsequent doses of $2.5 \mathrm{~g} / \mathrm{kg}$ ethanol $50 \%$ (ethanol-exposed group) or an equivalent volume of saline (control group) at $2 \mathrm{~h}$ intervals. Female with the same treatment were housed together and had negligible handling during the gestation.

\section{Blood alcohol concentration quantification}

Blood alcohol concentration associated with our preimplantation binge-like alcohol exposure paradigm was quantified over a $4 \mathrm{~h}$ period. To avoid supplementary stress on experimental animals, a different subset of pregnant E2.5 females $(n=12)$ was used for this experiment. Ethanol-exposed females $(n=3)$ were euthanized at each time point (1, 2, 3 and $4 \mathrm{~h}$ ), blood was collected, samples were centrifuged to separate serum and plasma, and alcohol was quantified using the EnzyChrome Ethanol Assay kit (BioAssays Systems/Cedarlane) following manufacturer's recommendations (1:5 plasma dilution).

\section{Morphological analysis}

At E10.5, pregnant females were euthanized, and embryos were collected and dissected for morphological evaluation using Leica stereo microscope. Using LasX software, measurements of the crown-rump length (top of the head to the end of the tail), occipital to nose length (occipital part of the head to the nasal process), height of the head (top of the head to the beginning of the torso) and length of the midbrain (occipital part to the midbrain/forebrain limit) were done. Similarly, morphological defects (e.g., severe developmental delay or growth restriction, brain or head malformation, heart anomaly or any other unexpected feature) were evaluated [108-110]. Three embryos $(n=3)$ revealed more than 2 morphological defects and were placed in the category of their main defect (delayed $n=2$, brain malformation $n=1$ ). Embryos that hatched ( $\mathrm{Ctrl} n=23$; EtOH $n=21$ ) from the yolk sac during dissection were excluded of both measurements and morphological analyses due to the possible deformation induced by the pressure on the embryo during the expulsion. The sex of each embryo was determined by qPCR using expression of $D d x 3$, on yolk sac DNA. Statistical analyses were done using GraphPad prism (version 8.4.3) for $t$ test with Welch correction and $f$-test for variance or R (version 3.5.0) for chi-square and proportional $z$-test. 


\section{Histological analysis and immunostaining}

Following collection, whole E10.5 embryos were fixed in $4 \%$ paraformaldehyde (PFA), post-fixed in $\mathrm{EtOH} 70 \%$ for $48 \mathrm{~h}$ and paraffin embedded [111]. Whole embryos were sectioned at $5 \mu \mathrm{M}$ and corresponding sections were stained with hematoxylin and eosin [112] to determine gross morphology, or for Cleaved Caspase-3 (Cell Signaling \#9579S) or Ki67 (Abcam \# AB15580) following manufacturer's protocol and counterstained with hematoxylin. Imaging was done using Zeiss Zen Axioscan Slide Scanner system. Images processing and quantification were performed using ImageJ. Three similar sub regions of forebrain and midbrain $\left(0.02 \mathrm{~mm}^{2}\right.$ each) were quantified across all samples. Statistical analyses were done using GraphPad prism (version 8.4.3) for $t$ test with Welch correction.

\section{DNA extraction and reduced representation bisulfite sequencing}

Following morphological analyses, embryonic E10.5 forebrains were isolated, flash frozen and kept at $-80{ }^{\circ} \mathrm{C}$. Genomic DNA was extracted from forebrains using the QIAamp DNA Micro kit (Qiagen \#56304) following manufacturer's recommendations. Extracted DNA was quantified using QuBit fluorimeter apparel with the Broad range DNA assay kit (ThermoFisher \#Q32853). The sex of each DNA sample was again validated by $D d x 3$ qPCR. DNA samples from forebrains with no apparent morphological defects were randomly selected, using 6 control embryos ( 3 males and 3 females, from 3 different litters) and 16 ethanol-exposed embryos ( 8 males and 8 females, from 6 different litters). EtOH-exposed and control embryo groups were similar in size and morphology (Additional file 1: Fig. S2). Genomic DNA was used to produce rapid Reduced Representation Bisulfite Sequencing (rRRBS) libraries as previously described [27, 113-116]. Briefly, 500 ng of DNA was digested with Msp1 restriction enzyme, adapters were attached to DNA fragments followed by sodium bisulfite conversion and amplification/indexation of libraries. Libraries were quantified using QuBit fluorimeter apparel with the High Sensitivity DNA assay kit (ThermoFisher \#Q32854). Quality control was assessed using BioAnalyzer and paired-end sequencing was done on Illumina HiSeq 2500 at the Genome Québec core facility. We obtained between 19 and $41 \mathrm{M}$ raw reads for each sample (Additional file 1: Table S1).

\section{Bioinformatics analysis}

Data processing, alignment ( $\mathrm{mm} 10$ genome) and methylation calls were performed using our established pipeline $[27,114-116]$ which includes tools such as Trim Galore (version 0.3.3) [117], BSMAP (version 2.90) [118] and R (version 3.5.0) (Additional file 1: Table S1). Differentially methylated regions were obtained with MethylKit (version 1.8.1) [119] using the Benjamini-Hochberg false discovery rate (FDR) procedure. Fixed parameters were used, including $100 \mathrm{bp}$ stepwise tiling windows, a minimum of 2 CpGs per tile and a threshold of $q<0.01$. DNA methylation level is calculated as the average methylation of all $\mathrm{CpGs}$ within a tile for all the samples within a condition (Fig. 3: minimum 5 samples/condition, $\geq 10 \times$ sequencing depth; Figs. 4, 5, 6 \& Additional file 1: Figs. S6-S7: minimum 3 samples/condition/sex, $\geq 10 \times$ sequencing depth). The number of $\mathrm{CpGs}$ per tile and bisulfite conversion rate (>97\%) were obtained using a custom Perl script.

Annotation of the analysed tiles was done using Homer (version 4.10.1) with mm10 reference genome. Gene ontology enrichment analysis was performed with Metascape [120] online tool using only differentially methylated tiles located in genic regions. Repeats and $\mathrm{CpG}$ islands coordinates were obtained from UCSC table browser databases (mm10 genome). CpG context tracks ( $\mathrm{CpG}$ shores and $\mathrm{CpG}$ shelves) were built by adding up respectively $0-2 \mathrm{~kb}$ and $2-4 \mathrm{~kb}$ from the $\mathrm{CpG}$ islands coordinates as previously describes $[114,121]$.

\section{RNA extraction and expression analysis by quantitative PCR (qPCR)}

Quantitative gene expression analyses were performed as previously [122, 123]. Briefly, embryonic E10.5 forebrains were isolated, flash frozen and kept at $-80{ }^{\circ} \mathrm{C}$ until RNA extraction. RNA was extracted using RNeasy Mini kit (Qiagen \#74004) following manufacturer's recommendations. Extracted RNA was quantified using QuBit fluorimeter apparel with the High Sensitivity RNA assay kit (ThermoFisher \#Q32852). 600 ng of RNA was used for cDNA conversion using SuperScript IV Reverse Transcriptase (ThermoFisher \#18090010). For each gene (primer sequence Additional file 1: Table S2), qPCR reactions were performed in triplicate on $5 \mathrm{ng}$ of cDNA using SensiFAST SYBR No-ROX (Bioline \#BIO98005) on a LightCycler 96 (Roche Life Science). Gene expression analysis and normalization was done using the $2^{-\Delta \Delta \mathrm{Ct}}$ method using Hprt1 and Pgk1 as reference genes. Statistical analyses were done using GraphPad prism (version 8.4.3) for $t$ test with Welch correction.

\section{Supplementary Information}

The online version contains supplementary material available at https://doi. org/10.1186/s13148-021-01151-0.

Additional file 1. Supplementary figures and tables. 


\section{Acknowledgements}

We thank the McGraw lab for critical comments and suggestions, as well as Elizabeth Maurice-Elder for editing.

\section{Authors' contributions}

$L M L$ and $S M$ conceptualized the study. $L M L$ and $M B L$ contributed in data acquisition. $L M L, K D, A L A, A L, M C$, LJM and DS participated in data analysis. $\mathrm{LML}, \mathrm{KD}$ and SM wrote the manuscript. All authors read and approved the final manuscript.

\section{Funding}

This work was supported by a research grant to SM from the SickKids Foundation and Fonds de Recherche du Québec en Santé (FRQS). LML is supported by Canadian Institutes of Health Research (CIHR) scholarship. MBL and KD are supported by FRQS scholarship/fellowship. ALA is supported by Université de Montréal and Réseau Québécois en Reproduction (RQR) scholarships. AL is supported by Université de Montréal and Centre de Recherche en Reproduction et Fertilité (CRRF) scholarships. SM is supported by FRQS - Junior 2 salary award.

\section{Availability of data and materials}

The data from this study have been submitted to the Gene Expression Omnibus (GSE162765).

\section{Declarations}

\section{Consent for publication}

Not applicable.

\section{Competing interests}

The authors declare that they have no competing interests.

\section{Author details}

${ }^{1} \mathrm{CHU}$ Sainte-Justine Research Center, 3175 Chemin de La Côte-Sainte-Catherine, Montréal, QC H3T 1C5, Canada. ${ }^{2}$ Department of Biochemistry and Molecular Medicine, Université de Montréal, 2900 Boulevard Edouard-Montpetit, Montréal, QC H3T 1 J4, Canada. ${ }^{3}$ McGill University Health Centre Glen Site, 1001 Boulevard Décarie, Montréal, QC H4A 3J1, Canada. ${ }^{4}$ Department of Pediatrics, McGill University, 1001 Boulevard Décarie, Montréal, QC H4A 3J1, Canada. ${ }^{5}$ Department of Pediatrics, Université de Montréal, 2900 Boulevard Edouard-Montpetit, Montréal, QC H3T 1 J4, Canada. ${ }^{6}$ Department of Obstetrics and Gynecology, Université de Montréal, 2900 Boulevard Edouard-Montpetit, Montréal, QC H3T 1J4, Canada.

Received: 4 January 2021 Accepted: 11 August 2021

Published online: 23 August 2021

\section{References}

1. May PA, Baete A, Russo J, Elliott AJ, Blankenship J, Kalberg WO, et al. Prevalence and characteristics of fetal alcohol spectrum disorders. Pediatrics. 2014;134(5):855-66.

2. Welch-Carre E. The neurodevelopmental consequences of prenatal alcohol exposure. Adv Neonatal Care. 2005;5(4):217-29.

3. Cook JL, Green CR, Lilley CM, Anderson SM, Baldwin ME, Chudley AE, et al. Fetal alcohol spectrum disorder: a guideline for diagnosis across the lifespan. CMAJ. 2016;188(3):191-7.

4. Legault LM, Bertrand-Lehouillier V, McGraw S. Pre-implantation alcohol exposure and developmental programming of FASD: an epigenetic perspective. Biochem Cell Biol. 2018;96(2):117-30.

5. Lange S, Probst C, Gmel G, Rehm J, Burd L, Popova S. Global prevalence of fetal alcohol spectrum disorder among children and youth: a systematic review and meta-analysis. JAMA Pediatr. 2017;171(10):948-56.

6. Popova S, Lange S, Probst C, Gmel G, Rehm J. Estimation of national, regional, and global prevalence of alcohol use during pregnancy and fetal alcohol syndrome: a systematic review and meta-analysis. Lancet Glob Health. 2017;5(3):e290-9.
7. Dwyer-Lindgren L, Flaxman AD, Ng M, Hansen GM, Murray CJ, Mokdad AH. Drinking patterns in US counties from 2002 to 2012. Am J Public Health. 2015;105(6):1120-7.

8. Grucza RA, Norberg K, Bucholz KK, Bierut LJ. Correspondence between secular changes in alcohol dependence and age of drinking onset among women in the United States. Alcohol Clin Exp Res. 2008;32(8):1493-501.

9. Lim SS, Vos T, Flaxman AD, Danaei G, Shibuya K, Adair-Rohani H, et al. A comparative risk assessment of burden of disease and injury attributable to 67 risk factors and risk factor clusters in 21 regions, 1990-2010: a systematic analysis for the Global Burden of Disease Study 2010. Lancet. 2012;380(9859):2224-60.

10. Popova S, Lange S, Probst C, Parunashvili N, Rehm J. Prevalence of alcohol consumption during pregnancy and Fetal Alcohol Spectrum Disorders among the general and Aboriginal populations in Canada and the United States. Eur J Med Genet. 2016.

11. Popova S, Lange S, Probst C, Shield K, Kraicer-Melamed H, FerreiraBorges C, et al. Actual and predicted prevalence of alcohol consumption during pregnancy in the WHO African Region. Trop Med Int Health. 2016;21(10):1209-39.

12. Tan $\mathrm{CH}$, Denny $\mathrm{CH}$, Cheal NE, Sniezek JE, Kanny D. Alcohol use and binge drinking among women of childbearing age-United States, 2011-2013. MMWR Morb Mortal Wkly Rep. 2015;64(37):1042-6.

13. Thomas G. Levels and patterns of alcohol use in Canada. Alcohol Price Policy Series. Report 1. Ottawa, ON: Canadian Centre on Substance Abuse. 2012; http://www.ccsa.ca/Resource\%20Library/CCSA-PatternsAlcohol-Use-Policy-Canada-2012-en.pdf.

14. Wilsnack SC, Wilsnack RW, Kantor LW. Focus on: women and the costs of alcohol use. Alcohol Res. 2013;35(2):219-28.

15. Sedgh G, Singh S, Hussain R. Intended and unintended pregnancies worldwide in 2012 and recent trends. Stud Fam Plann. 2014;45(3):301-14.

16. Öztürk NC, Resendiz M, Öztürk H, Zhou FC. DNA Methylation program in normal and alcohol-induced thinning cortex. Alcohol. 2017:60:135-47.

17. Garro AJ, McBeth DL, Lima V, Lieber CS. Ethanol consumption inhibits fetal DNA methylation in mice: implications for the fetal alcohol syndrome. Alcohol Clin Exp Res. 1991;15(3):395-8.

18. Chater-Diehl EJ, Laufer BI, Castellani CA, Alberry BL, Singh SM. Alteration of gene expression, DNA methylation, and histone methylation in free radical scavenging networks in adult mouse hippocampus following fetal alcohol exposure. PLoS ONE. 2016;11(5):e0154836.

19. Laufer BI, Kapalanga J, Castellani CA, Diehl EJ, Yan L, Singh SM. Associative DNA methylation changes in children with prenatal alcohol exposure. Epigenomics. 2015;7(8):1259-74.

20. McGraw S, Oakes CC, Martel J, Cirio MC, de Zeeuw P, Mak W, et al. Loss of DNMT10 disrupts imprinted X chromosome inactivation and accentuates placental defects in females. PLoS Genet. 2013;9(11):e1003873.

21. Breton-Larrivée M, Elder E, McGraw S. DNA methylation, environmental exposures and early embryo development. Anim Reprod. 2019;16(3):465-74.

22. McGraw S, Trasler IM. Oocyte epigenetics and the risks for imprinting disorders associated with assisted reproduction. Biol Pathol Oocyte Role Fert Med Nuclear Reprogram. 2013:384.

23. Zhu P, Guo H, Ren Y, Hou Y, Dong J, Li R, et al. Single-cell DNA methylome sequencing of human preimplantation embryos. Nat Genet. 2018;50(1):12-9.

24. Farthing CR, Ficz G, Ng RK, Chan CF, Andrews S, Dean W, et al. Global mapping of DNA methylation in mouse promoters reveals epigenetic reprogramming of pluripotency genes. PLoS Genet. 2008;4(6):e1000116.

25. Oswald J, Engemann S, Lane N, Mayer W, Olek A, Fundele R, et al. Active demethylation of the paternal genome in the mouse zygote. Curr Biol. 2000;10(8):475-8.

26. Peat JR, Dean W, Clark SJ, Krueger F, Smallwood SA, Ficz G, et al. Genome-wide bisulfite sequencing in zygotes identifies demethylation targets and maps the contribution of TET3 oxidation. Cell Rep. 2014;9(6):1990-2000.

27. McGraw S, Zhang JX, Farag M, Chan D, Caron M, Konermann C, et al. Transient DNMT1 suppression reveals hidden heritable marks in the genome. Nucleic Acids Res. 2015;43(3):1485-97. 
28. ReikW, Dean W, Walter J. Epigenetic reprogramming in mammalian development. Science. 2001;293(5532):1089-93.

29. Messerschmidt DM, Knowles BB, Solter D. DNA methylation dynamics during epigenetic reprogramming in the germline and preimplantation embryos. Genes Dev. 2014;28(8):812-28.

30. Kazakevych J, Sayols S, Messner B, Krienke C, Soshnikova N. Dynamic changes in chromatin states during specification and differentiation of adult intestinal stem cells. Nucleic Acids Res. 2017;45(10):5770-84.

31. Liu J, Banerjee A, Herring CA, Attalla J, Hu R, Xu Y, et al. Neurog3independent methylation is the earliest detectable mark distinguishing pancreatic progenitor identity. Dev Cell. 2019;48(1):49-63.e7.

32. Sanosaka T, Imamura T, Hamazaki N, Chai M, Igarashi K, Ideta-Otsuka $M$, et al. DNA methylome analysis identifies transcription factor-based epigenomic signatures of multilineage competence in neural stem/ progenitor cells. Cell Rep. 2017;20(12):2992-3003.

33. Zhang Y, Xiang Y, Yin Q, Du Z, Peng X, Wang Q, et al. Dynamic epigenomic landscapes during early lineage specification in mouse embryos. Nat Genet. 2018;50(1):96-105.

34. Pérez-Cerezales S, Ramos-lbeas P, Rizos D, Lonergan P, Bermejo-Alvarez P, Gutiérrez-Adán A. Early sex-dependent differences in response to environmental stress. Reproduction. 2018;155(1):R39-r51.

35. Bermejo-Alvarez P, Rizos D, Lonergan P, Gutierrez-Adan A. Transcriptional sexual dimorphism during preimplantation embryo development and its consequences for developmental competence and adult health and disease. Reproduction. 2011;141(5):563-70.

36. Donjacour A, Liu X, Lin W, Simbulan R, Rinaudo PF. In vitro fertilization affects growth and glucose metabolism in a sex-specific manner in an outbred mouse model. Biol Reprod. 2014;90(4):80.

37. Lowe R, Gemma C, Rakyan VK, Holland ML. Sexually dimorphic gene expression emerges with embryonic genome activation and is dynamic throughout development. BMC Genomics. 2015;16(1):295.

38. Miozzo F, Arnould H, de Thonel A, Schang AL, Sabéran-Djoneidi D, Baudry A, et al. Alcohol exposure promotes DNA methyltransferase DNMT3A upregulation through reactive oxygen species-dependent mechanisms. Cell Stress Chaperones. 2018;23(1):115-26.

39. Wu D, Cederbaum Al. Alcohol, oxidative stress, and free radical damage. Alcohol Res Health. 2003;27(4):277-84.

40. Mandal C, Halder D, Jung KH, Chai YG. Gestational alcohol exposure altered DNA methylation status in the developing fetus. Int J Mol Sci. 2017;18(7).

41. Haycock PC, Ramsay M. Exposure of mouse embryos to ethanol during preimplantation development: effect on DNA methylation in the h19 imprinting control region. Biol Reprod. 2009;81(4):618-27.

42. Patten AR, Fontaine CJ, Christie BR. A comparison of the different animal models of fetal alcohol spectrum disorders and their use in studying complex behaviors. Front Pediatr. 2014;2:93.

43. Perkins A, Lehmann C, Lawrence RC, Kelly SJ. Alcohol exposure during development: impact on the epigenome. Int J Dev Neurosci. 2013;31(6):391-7.

44. Boschen KE, Criss KJ, PalamarchoukV, Roth TL, Klintsova AY. Effects of developmental alcohol exposure vs. intubation stress on BDNF and TrkB expression in the hippocampus and frontal cortex of neonatal rats. Int J Dev Neurosci. 2015:43:16-24

45. Mantha K, Laufer Bl, Singh SM. Molecular changes during neurodevelopment following second-trimester binge ethanol exposure in a mouse model of fetal alcohol spectrum disorder: from immediate effects to long-term adaptation. Dev Neurosci. 2014;36(1):29-43.

46. Abel E. Effects of ethanol on pregnant rats and their offspring. Psychopharmacology. 1978:57(1):5-11.

47. Sulik KK, Johnston MC, Webb MA. Fetal alcohol syndrome: embryogenesis in a mouse model. Science. 1981;214(4523):936-8.

48. El Shawa H, Abbott CW 3rd, Huffman KJ. Prenatal ethanol exposure disrupts intraneocortical circuitry, cortical gene expression, and behavior in a mouse model of FASD. J Neurosci. 2013;33(48):18893-905.

49. Ghimire SR, Dhungel S, Rai D, Jha CB, Saxena AK, Maskey D. Effect of prenatal exposure of alcohol in the morphology of developing rat embryo. Nepal Med Coll J. 2008;10(1):38-40.

50. Le TN, Zhou QP, Cobos I, Zhang S, Zagozewski J, Japoni S, et al. GABAergic interneuron differentiation in the basal forebrain is mediated through direct regulation of glutamic acid decarboxylase isoforms by dlx homeobox transcription factors. J Neurosci. 2017;37(36):8816-29.
51. Lim L, Mi D, Llorca A, Marín O. Development and functional diversification of cortical interneurons. Neuron. 2018;100(2):294-313.

52. Sussel L, Marin O, Kimura S, Rubenstein JL. Loss of Nkx21 homeobox gene function results in a ventral to dorsal molecular respecification within the basal telencephalon: evidence for a transformation of the pallidum into the striatum. Development. 1999;126(15):3359-70.

53. Nóbrega-Pereira S, Kessaris N, Du T, Kimura S, Anderson SA, Marín O. Postmitotic Nkx2-1 controls the migration of telencephalic interneurons by direct repression of guidance receptors. Neuron. 2008;59(5):733-45.

54. Alzu'bi A, Lindsay S, Kerwin J, Looi SJ, Khalil F, Clowry GJ. Distinct cortical and sub-cortical neurogenic domains for GABAergic interneuron precursor transcription factors NKX2.1, OLIG2 and COUP-TFII in early fetal human telencephalon. Brain Struct Funct. 2017;222(5):2309-28.

55. Kitamura K, Yanazawa M, Sugiyama N, Miura H, lizuka-Kogo A, Kusaka $M$, et al. Mutation of ARX causes abnormal development of forebrain and testes in mice and X-linked lissencephaly with abnormal genitalia in humans. Nat Genet. 2002;32(3):359-69.

56. Colasante G, Collombat P, Raimondi V, Bonanomi D, Ferrai C, Maira $M$, et al. Arx is a direct target of Dlx2 and thereby contributes to the tangential migration of GABAergic interneurons. J Neurosci. 2008;28(42):10674-86.

57. Lunde ER, Washburn SE, Golding MC, Bake S, Miranda RC, Ramadoss J. Alcohol-induced developmental origins of adult-onset diseases. Alcohol Clin Exp Res. 2016;40(7):1403-14.

58. Chang RC, Skiles WM, Chronister SS, Wang H, Sutton GI, Bedi YS, et al. DNA methylation-independent growth restriction and altered developmental programming in a mouse model of preconception male alcohol exposure. Epigenetics. 2017;12(10):841-53.

59. Laufer BI, Chater-Diehl EJ, Kapalanga J, Singh SM. Long-term alterations to DNA methylation as a biomarker of prenatal alcohol exposure: from mouse models to human children with fetal alcohol spectrum disorders. Alcohol. 2017;60:67-75.

60. Lussier AA, Morin AM, Maclsaac JL, Salmon J, Weinberg J, Reynolds JN, et al. DNA methylation as a predictor of fetal alcohol spectrum disorder. Clin Epigenetics. 2018;10:5.

61. Portales-Casamar E, Lussier AA, Jones MJ, Maclsaac JL, Edgar RD, Mah $\mathrm{SM}$, et al. DNA methylation signature of human fetal alcohol spectrum disorder. Epigenetics Chromatin. 2016;9:25.

62. Petrelli B, Weinberg J, Hicks GG. Effects of prenatal alcohol exposure (PAE): insights into FASD using mouse models of PAE. Biochem Cell Biol. 2018:96(2):131-47.

63. Liyanage VR, Zachariah RM, Davie JR, Rastegar M. Ethanol deregulates Mecp2/MeCP2 in differentiating neural stem cells via interplay between 5-methylcytosine and 5-hydroxymethylcytosine at the Mecp2 regulatory elements. Exp Neurol. 2015;265:102-17.

64. Wiebold JL, Becker WC. In-vivo and in-vitro effects of ethanol on mouse preimplantation embryos. J Reprod Fertil. 1987;80(1):49-57.

65. Abel EL. Prenatal effects of alcohol on growth: a brief overview. Fed Proc. 1985;44(7):2318-22.

66. Padmanabhan R, Hameed MS. Effects of acute doses of ethanol administered at pre-implantation stages on fetal development in the mouse. Drug Alcohol Depend. 1988;22(1-2):91-100.

67. Sandor S, Gârban Z, Checiu M, Daradics L. The presence of ethanol in the oviductal and uterine luminal fluids of alcoholized rats. Morphol Embryol (Bucur). 1981;27(4):303-9.

68. Pagé-Larivière F, Campagna C, Sirard MA. Mechanisms involved in porcine early embryo survival following ethanol exposure. Toxicol Sci. 2017;156(1):289-99.

69. Mustapha TA, Chang RC, Garcia-Rhodes D, Pendleton D, Johnson NM, Golding MC. Gestational exposure to particulate air pollution exacerbates the growth phenotypes induced by preconception paternal alcohol use: a multiplex model of exposure. Environ Epigenet. 2020;6(1):1011.

70. Chang RC, Thomas KN, Bedi YS, Golding MC. Programmed increases in LXRa induced by paternal alcohol use enhance offspring metabolic adaptation to high-fat diet induced obesity. Mol Metab. 2019:30:161-72.

71. Bedi Y, Chang RC, Gibbs R, Clement TM, Golding MC. Alterations in sperm-inherited noncoding RNAs associate with late-term fetal growth 
restriction induced by preconception paternal alcohol use. Reprod Toxicol. 2019;87:11-20.

72. Chang RC, Wang H, Bedi Y, Golding MC. Preconception paternal alcohol exposure exerts sex-specific effects on offspring growth and long-term metabolic programming. Epigenetics Chromatin. 2019;12(1):9.

73. Cirio MC, Martel J, Mann M, Toppings M, Bartolomei M, Trasler J, et al. DNA methyltransferase 10 functions during preimplantation development to preclude a profound level of epigenetic variation. Dev Biol. 2008;324(1):139-50

74. Toppings M, Castro C, Mills PH, Reinhart B, Schatten G, Ahrens ET, et al. Profound phenotypic variation among mice deficient in the maintenance of genomic imprints. Hum Reprod. 2008;23(4):807-18.

75. Cirio MC, Ratnam S, Ding F, Reinhart B, Navara C, Chaillet JR. Preimplantation expression of the somatic form of Dnmt1 suggests a role in the inheritance of genomic imprints. BMC Dev Biol. 2008;8:9.

76. Dasmahapatra AK, Khan IA. DNA methyltransferase expressions in Japanese rice fish (Oryzias latipes) embryogenesis is developmentally regulated and modulated by ethanol and 5-azacytidine. Comp Biochem Physiol C Toxicol Pharmacol. 2015;176-177:1-9.

77. Kindsfather AJ, Czekalski MA, Pressimone CA, Erisman MP, Mann MRW. Perturbations in imprinted methylation from assisted reproductive technologies but not advanced maternal age in mouse preimplantation embryos. Clin Epigenetics. 2019;1 1(1):162.

78. Market-Velker BA, Zhang L, Magri LS, Bonvissuto AC, Mann MR. Dual effects of superovulation: loss of maternal and paternal imprinted methylation in a dose-dependent manner. Hum Mol Genet. 2010;19(1):36-51.

79. Ho-Shing O, Dulac C. Influences of genomic imprinting on brain function and behavior. Curr Opin Behav Sci. 2019;25:66-76.

80. Seisenberger S, Peat JR, Hore TA, Santos F, Dean W, Reik W. Reprogramming DNA methylation in the mammalian life cycle: building and breaking epigenetic barriers. Philos Trans R Soc Lond B Biol Sci. 2013;368(1609):20110330.

81. Whidden L, Martel J, Rahimi S, Chaillet JR, Chan D, Trasler JM. Compromised oocyte quality and assisted reproduction contribute to sexspecific effects on offspring outcomes and epigenetic patterning. Hum Mol Genet. 2016;25(21):4649-60.

82. Tan $K$, Wang Z, Zhang Z, An L, Tian J. IVF affects embryonic development in a sex-biased manner in mice. Reproduction. 2016;151(4):443-53.

83. Skuse DH. Imprinting, the X-chromosome, and the male brain: explaining sex differences in the liability to autism. Pediatric Res. 2000;47(1):9.

84. Hart R, Norman RJ. The longer-term health outcomes for children born as a result of IVF treatment: part I-General health outcomes. Hum Reprod Update. 2013;19(3):232-43.

85. Källén B, Finnström O, Lindam A, Nilsson E, Nygren KG, Olausson PO. Cancer risk in children and young adults conceived by in vitro fertilization. Pediatrics. 2010;126(2):270-6.

86. Kenton JA, Castillo VK, Kehrer PE, Brigman JL. Moderate prenatal alcohol exposure impairs visual-spatial discrimination in a sex-specific manner: effects of testing order and difficulty on learning performance. Alcohol Clin Exp Res. 2020.

87. de Ávila MAP, Gonçalves RM, Nascimento ECC, Cabral LDM, Vilela FC, Giusti-Paiva A. Prenatal exposure to alcohol impairs social play behavior in adolescent male mice. Neurotoxicology. 2020;79:142-9.

88. Petropoulos S, Edsgärd D, Reinius B, Deng Q, Panula SP, Codeluppi S, et al. Single-cell RNA-Seq reveals lineage and $X$ chromosome dynamics in human preimplantation embryos. Cell. 2016;165(4):1012-26.

89. Bermejo-Alvarez P, Rizos D, Rath D, Lonergan P, Gutierrez-Adan A. Epigenetic differences between male and female bovine blastocysts produced in vitro. Physiol Genomics. 2008;32(2):264-72.

90. Bermejo-Alvarez P, Rizos D, Rath D, Lonergan P, Gutierrez-Adan A. Sex determines the expression level of one third of the actively expressed genes in bovine blastocysts. Proc Natl Acad Sci USA. 2010;107(8):3394-9.

91. Gebert C, Wrenzycki C, Herrmann D, Gröger D, Thiel J, Reinhardt R, et al. DNA methylation in the IGF2 intragenic DMR is re-established in a sexspecific manner in bovine blastocysts after somatic cloning. Genomics. 2009;94(1):63-9.
92. Terasaki LS, Gomez J, Schwarz JM. An examination of sex differences in the effects of early-life opiate and alcohol exposure. Philos Trans R Soc Lond B Biol Sci. 2016:371(1688):20150123.

93. Hernández-Miranda LR, Parnavelas JG, Chiara F. Molecules and mechanisms involved in the generation and migration of cortical interneurons. ASN Neuro. 2010;2(2):e00031.

94. Yun K, Fischman S, Johnson J, Hrabe de Angelis M, Weinmaster G, Rubenstein JL. Modulation of the notch signaling by Mash1 and DIx $1 / 2$ regulates sequential specification and differentiation of progenitor cell types in the subcortical telencephalon. Development. 2002;129(21):5029-40.

95. Anderson SA, Eisenstat DD, Shi L, Rubenstein JL. Interneuron migration from basal forebrain to neocortex: dependence on Dlx genes. Science. 1997:278(5337):474-6.

96. Cobos I, Borello U, Rubenstein JL. Dlx transcription factors promote migration through repression of axon and dendrite growth. Neuron. 2007;54(6):873-88.

97. Friocourt G, Parnavelas JG. Identification of Arx targets unveils new candidates for controlling cortical interneuron migration and differentiation. Front Cell Neurosci. 2011;5:28.

98. Bird CW, Taylor DH, Pinkowski NJ, Chavez GJ, Valenzuela CF. Long-term reductions in the population of GAB aergic interneurons in the mouse hippocampus following developmental ethanol exposure. Neuroscience. 2018;383:60-73.

99. Cunningham LA, Newville J, Li L, Tapia P, Allan AM, Valenzuela CF. Prenatal alcohol exposure leads to enhanced serine 9 phosphorylation of glycogen synthase kinase-3 3 (GSK-3 $\beta$ ) in the hippocampal dentate gyrus of adult mouse. Alcohol Clin Exp Res. 2017;41(11):1907-16.

100. Larsen ZH, Chander P, Joyner JA, Floruta CM, Demeter TL, Weick JP. Effects of ethanol on cellular composition and network excitability of human pluripotent stem cell-derived neurons. Alcohol Clin Exp Res. 2016;40(11):2339-50.

101. Cuzon VC, Yeh PW, Yanagawa Y, Obata K, Yeh HH. Ethanol consumption during early pregnancy alters the disposition of tangentially migrating GABAergic interneurons in the fetal cortex. J Neurosci. 2008;28(8):1854-64.

102. Skorput AG, Lee SM, Yeh PW, Yeh HH. The NKCC1 antagonist bumetanide mitigates interneuronopathy associated with ethanol exposure in utero. Elife. 2019;8.

103. Paterno R, Casalia M, Baraban SC. Interneuron deficits in neurodevelopmental disorders: implications for disease pathology and interneuronbased therapies. Eur J Paediatr Neurol. 2020;24:81-8.

104. Rossignol E. Genetics and function of neocortical GABAergic interneurons in neurodevelopmental disorders. Neural Plast. 2011;2011:649325.

105. Skorput AG, Gupta VP, Yeh PW, Yeh HH. Persistent interneuronopathy in the prefrontal cortex of young adult offspring exposed to ethanol in utero. J Neurosci. 2015;35(31):10977-88.

106. Kleiber ML, Mantha K, Stringer RL, Singh SM. Neurodevelopmental alcohol exposure elicits long-term changes to gene expression that alter distinct molecular pathways dependent on timing of exposure. J Neurodev Disord. 2013;5(1):6.

107. Burgess DJ, Moritz KM. Prenatal alcohol exposure and developmental programming of mental illness. J Dev Orig Health Dis. 2020;11(3):211-21.

108. Christensen KE, Hou W, Bahous RH, Deng L, Malysheva OV, Arning E, et al. Moderate folic acid supplementation and MTHFD1-synthetase deficiency in mice, a model for the $\mathrm{R} 653 \mathrm{Q}$ variant, result in embryonic defects and abnormal placental development. Am J Clin Nutr. 2016;104(5):1459-69.

109. Christensen KE, Deng L, Leung KY, Arning E, Bottiglieri T, Malysheva $\mathrm{OV}$, et al. A novel mouse model for genetic variation in 10-formyltetrahydrofolate synthetase exhibits disturbed purine synthesis with impacts on pregnancy and embryonic development. Hum Mol Genet. 2013:22(18):3705-19.

110. Theiler K. The house mouse: atlas of embryonic development. Berlin: Springer; 2013

111. Li L, Jayabal S, Ghorbani M, Legault LM, McGraw S, Watt AJ, et al. ATAT1 regulates forebrain development and stress-induced tubulin hyperacetylation. Cell Mol Life Sci. 2019;76(18):3621-40. 
112. Shaffer B, McGraw S, Xiao SC, Chan D, Trasler J, Chaillet JR. The DNMT1 intrinsically disordered domain regulates genomic methylation during development. Genetics. 2015;199(2):533-41.

113. Legault L-M, Chan D, McGraw S. Rapid multiplexed reduced representation bisulfite sequencing library prep (rRRBS). Bio-Protoc. 2019;9:4

114. Legault LM, Doiron K, Lemieux A, Caron M, Chan D, Lopes FL, et al. Developmental genome-wide DNA methylation asymmetry between mouse placenta and embryo. Epigenetics. 2020;15(8):800-15.

115. Pierre WC, Legault LM, Londono I, McGraw S, Lodygensky GA. Alteration of the brain methylation landscape following postnatal inflammatory injury in rat pups. Faseb J. 2020;34(1):432-45.

116. Piché J, Gosset N, Legault LM, Pacis A, Oneglia A, Caron M, et al. Molecular signature of CAID syndrome: noncanonical roles of SGO1 in regulation of TGF- $\beta$ signaling and epigenomics. Cell Mol Gastroentero Hepatol. 2019;7(2):411-31.

117. Krueger F. Trim Galore: a wrapper tool around Cutadapt and FastQC to consistently apply quality and adapter trimming to FastQ files, with some extra functionality for Mspl-digested RRBS-type (Reduced Representation Bisufite-Seq) libraries. http://www.bioinformaticsbabrah amacuk/projects/trim galore/. Date of access: 28 Apr 2016. 2012

118. Xi Y, Li W. BSMAP: whole genome bisulfite sequence MAPping program. BMC Bioinform. 2009:10:232.
119. Akalin A, Kormaksson M, Li S, Garrett-Bakelman FE, Figueroa ME, Melnick $A$, et al. methylKit: a comprehensive $R$ package for the analysis of genome-wide DNA methylation profiles. Genome Biol. 2012;13(10):R87.

120. Zhou Y, Zhou B, Pache L, Chang M, Khodabakhshi AH, Tanaseichuk O, et al. Metascape provides a biologist-oriented resource for the analysis of systems-level datasets. Nat Commun. 2019;10(1):1523.

121. Bibikova M, Barnes B, Tsan C, Ho V, Klotzle B, Le JM, et al. High density DNA methylation array with single CpG site resolution. Genomics. 2011:98(4):288-95

122. Da Costa EM, Armaos G, McInnes G, Beaudry A, Moquin-Beaudry G, Bertrand-Lehouillier $\mathrm{V}$, et al. Heart failure drug proscillaridin A targets MYC overexpressing leukemia through global loss of lysine acetylation. J Exp Clin Cancer Res. 2019;38(1):251.

123. McGraw S, Vigneault C, Sirard MA. Temporal expression of factors involved in chromatin remodeling and in gene regulation during early bovine in vitro embryo development. Reproduction. 2007;133(3):597-608.

\section{Publisher's Note}

Springer Nature remains neutral with regard to jurisdictional claims in published maps and institutional affiliations.
Ready to submit your research? Choose BMC and benefit from:

- fast, convenient online submission

- thorough peer review by experienced researchers in your field

- rapid publication on acceptance

- support for research data, including large and complex data types

- gold Open Access which fosters wider collaboration and increased citations

- maximum visibility for your research: over $100 \mathrm{M}$ website views per year

At BMC, research is always in progress.

Learn more biomedcentral.com/submissions 